\title{
L'ETHNOLOGIE MUSICALE SELON ANDRÉ SCHAEFFNER, ENTRE MUSÉE ET PERFORMANCE
}

\author{
Olivier Roueff
}

Ed. Sc. Humaines | Revue d'Histoire des Sciences Humaines

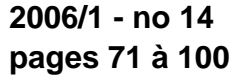

ISSN 1622-468X

Article disponible en ligne à l'adresse:

http://www.cairn.info/revue-histoire-des-sciences-humaines-2006-1-page-71.htm

Pour citer cet article :

Roueff Olivier , «L'ethnologie musicale selon André Schaeffner, entre musée et performance », Revue d'Histoire des Sciences Humaines, 2006/1 no 14, p. 71-100. DOI : 10.3917/rhsh.014.0071

Distribution électronique Cairn.info pour Ed. Sc. Humaines.

(c) Ed. Sc. Humaines. Tous droits réservés pour tous pays.

La reproduction ou représentation de cet article, notamment par photocopie, n'est autorisée que dans les limites des conditions générales d'utilisation du site ou, le cas échéant, des conditions générales de la licence souscrite par votre établissement. Toute autre reproduction ou représentation, en tout ou partie, sous quelque forme et de quelque manière que ce soit, est interdite sauf accord préalable et écrit de l'éditeur, en dehors des cas prévus par la législation en vigueur en France. II est précisé que son stockage dans une base de données est également interdit. 


\title{
L'ethnologie musicale selon André Schaeffner, entre musée et performance
}

\author{
Olivier ROUEFF
}

\begin{abstract}
Résumé
André Schaeffner a créé et dirigé le département d'ethnologie musicale du Musée d'ethnographie du Trocadéro (1929), puis du Musée de l'homme (1937-1964). L'article interroge la genèse des thèses rassemblées et systématisées dans la somme programmatique qu'il publie en 1936, Origine des instruments de musique. Introduction ethnologique à l'histoire de la musique instrumentale. En restituant ses expériences de formation, notamment au pôle savant de la nébuleuse intellectuelle catholique, et ses déplacements positionnels, du pôle scholiste puis néoclassique du champ musical jusqu'à la reconversion à l'ethnographie durkheimienne, il s'agit de rendre compte du caractère improbable de son organologie: tournée contre le graphocentrisme «philologique » de l'histoire de la musique et définissant la musique comme performance, elle reste néanmoins tributaire du dispositif de savoir muséal, centré sur la collecte et la classification d'objets. Elle vient ainsi paradoxalement refonder une philosophie essentialiste de la musique, durablement ancrée dans la dissociation «scolastique » entre codes musicaux (approchés par les transcriptions graphiques) et usages instrumentistes (renvoyant à l'inertie raciale ou culturelle des corps musiciens).
\end{abstract}

Mots-clés : Ethnologie et muséocentrisme - Musicologie et graphocentrisme - Genèse et dispositifs de savoirs.

\begin{abstract}
Between Museum and Performance : André Schaeffner's Musical Ethnology
André Schaeffner was the founder and director of the department of ethnomusicology at the successive Museums of anthropology in Paris from 1929 to 1964. His thesis were gathered and developed in his masterpiece published in 1936, Origine des instruments de musique. Introduction ethnologique à l'histoire de la musique instrumentale. We explain the singularity of this work by studying Schaeffner's intellectual socialisations, especially in the catholic circles he entered, and the plurality of his positions, from "scholism " to "neoclassicism ", as a musical journalist, and then to "durkheimian" ethnology. He defines music as a performance, against the "graphocentrism » of musicology, but remains dependant from the context of knowledge that the museum organizes, centered on gatherings and classifications of material objects. This leads him paradoxically to an essentialist philosophy of music, where musical codes (ie graphic transcriptions) and performers' habits (ie racial or cultural remanences of musicians' bodies) are dissociated.
\end{abstract}

Key-words : Ethnology and Museocentrism - Musicology and Graphocentrism - Formation and Contextes of Knowledge. 
«parce que c'est là où j'ai retrouvé l'Ancien Testament encore vivant $\gg^{1}$

La redéfinition de l'ethnologie dans l'entre-deux-guerres, autour du pôle durkheimien et d'institutions à la fois marginales dans le monde universitaire et soutenues par l'État (l'Institut d'ethnologie, les musées spécialisés, l'Encyclopédie française), a entre autres effets celui d'ouvrir un espace pour des programmes ethnographiques concernant les faits musicaux définis comme lointains (socialement ou culturellement) ${ }^{2}$. La description savante de pratiques musicales rurales ou exotiques était jusque-là produite essentiellement par des musicologues « folkloristes » d'un côté (Julien Tiersot en étant la principale figure) ${ }^{3}$, et de l'autre, par les diverses espèces de savants du monde colonial (voyageurs, administrateurs, ethnographes amateurs, premiers ethnologues...) ${ }^{4}$. Avec la création en 1929 d'un département d'organologie, puis d'ethnologie musicale en 1932, au sein du Musée d'ethnographie du Trocadéro (MET), transformé en Musée de l'homme en 1937 (le domaine folklorique étant alors pris en charge par un Musée des arts et traditions populaires (MATP) qui n'aura de locaux propres qu'en 1969) - c'est un secteur ethnographique qui se spécialise et s'institutionnalise, parvenant à monopoliser la légitimité scientifique pour l'investigation des faits musicaux exotiques.

Notre enquête interroge les sources et les pratiques de savoir élaborées par celui à qui fut confié le département d'ethnologie musicale du MET puis du Musée de l'homme - André Schaeffner (1895-1980) ${ }^{5}$. Nous montrerons que l'insertion de Schaeffner dans le champ musical, exclusive durant les années 1920 en tant que critique et historien, est demeurée prépondérante alors qu'il investissait le secteur ethnologique au tournant des années 1930, et y restait en position dominante (pour le domaine musical) jusqu'aux années 1960. Par là, nous ne désignons pas seulement sa double position - puisqu'il est demeuré une sorte de figure excentrée du champ musical ${ }^{6}$ - mais surtout les logiques des intérêts et des thèses qui organisent ses travaux ethnographiques. Cette particularité permet d'analyser les effets de la rencontre entre le « graphocentrisme» de la discipline musicologique et le « muséocentrisme » de la discipline ethnologique - autrement dit, entre un savoir centré sur l'objet «partition» et l'héritage des méthodes philologiques, et un savoir centré sur la collecte et la description des objets muséaux (comme les instruments de musique), selon les

\footnotetext{
${ }^{1}$ Réponse d'André Schaeffner à une question sur «la raison de ses attaches au continent noir » (GERGELY, 1980, 259).

${ }^{2}$ Nous remercions vivement Jacques Cheyronnaud et Jean Jamin pour les discussions, informations et documents qu'ils nous ont fournis, Rémy Campos pour sa relecture attentive et les améliorations sensibles qu'elle a suscitées, ainsi que Nicolas Donin, Frédérick Keck et les lecteurs anonymes pour leurs utiles suggestions. Nous restons seul responsable du propos et de ses faiblesses.

${ }^{3}$ CheyronnaUd, 1994 : Joseph d'Ortigue (1802-1866) est le premier à utiliser l'expression d' « ethnographie musicale », avant Amintore Galli (1845-1919) et Julien Tiersot (1857-1936) et ses Notes d'ethnographie musicale parues en 1900 (il traita aussi de musiques exotiques). Sur Tiersot, $c f$. CHEYRONNAUD, 1995.

${ }^{4}$ SIBEUD, 1992.

${ }^{5}$ Il est en effet peu évoqué dans les travaux qui, depuis une vingtaine d'années, documentent cette période de la discipline. Cf. CheyronNAUd, 1986 ; JAMIN, 1986 ; 1989 ; FAURE, 1989 ; FABRE, 1997 ; LEBOVICS, 1995 ; Sibeud, 1997 ; De L'Estoile, 2001 ; Rouget, 2001 ; Weber F., 2002 ; ChEYRONNAUd, 2003.

${ }^{6}$ Parmi les signes les plus visibles de cette position : l'organisation avec Denise Tual des Concerts de la Pléiade, de 1943 à 1947, qui programma les compositeurs français modernes et contemporains (PORCILE, 2001, 95); le dialogue avec Pierre Boulez et la rédaction des programmes et notices du Domaine musical dans ses premières années (à partir de 1954), autrement dit l'avant-garde sérielle qui s'inscrit dans la filiation atonale ; la vice-présidence de 1948 à 1958, puis la présidence de 1958 à 1968 de la Société française de musicologie ; la qualité de membre de l'Académie Charles Cros à partir de 1948.
} 
méthodes de classification dérivées des sciences naturelles. En important les perspectives élaborées comme critique musical anti-rationaliste et néoclassique, et en développant une muséologie contextualiste en collaboration avec Georges Henri Rivière, Schaeffner fait de cette rencontre un déplacement qu'on peut dire à la fois innovant et inabouti, tant en regard des conventions d'administration des preuves empiriques qui s'imposent alors dans la discipline que du projet qu'il élabore et met en avant. En effet, nous établirons qu'il fait jouer théoriquement le centrement sur le corps musicien et la performance musicale contre le privilège de l'objet muséal ou de la partition, mais demeure en pratique tributaire du dispositif de savoir muséal qu'il investit, et «sauve» ainsi, paradoxalement, ce que le graphocentrisme des savants du monde musical a contribué à instituer et à durcir au $\mathrm{XIX}^{\mathrm{e}}$ siècle : La Musique comme essence réifiée, existant en-deçà de ses actualisations.

\section{I - La médiation catholique}

André Schaeffner entre dans le métier musicographique ${ }^{7}$ au début des années 1920 par les réseaux lettrés catholiques. En effet, ses premières identifications intellectuelles appartiennent à la « nébuleuse intellectuelle catholique » qui se forme autour de la Première Guerre mondiale ${ }^{8}$. Elles le conduisent à investir les réseaux «scholistes» ou «d'indystes », autrement dit le pôle puriste dominant du champ musical, réactionnaire et moraliste en ce qui concerne ses principaux leaders, mais aussi à endosser la variante sensualiste, et non spiritualiste, de l'anti-rationalisme qu'ils partagent - orientation qui plus est développée par de nouvelles activités (la «rythmique», la musique ancienne, la musicologie savante, le jazz). Ce glissement suit le rapprochement des universitaires catholiques qu'il fréquente (les frères Baruzi, Gabriel Marcel) avec la Nouvelle Revue Française, qui se démarquent par là des conceptions hétéronomes de l'art promues par un Charles Maurras ou un Jacques Maritain. Il aboutit ainsi à l'investissement d'une position en quelque sorte opposée au sein de la jeune Revue Musicale - sa fraction moderniste et autonomiste (tout au moins dans les années 1920), liée à la NRF. L'explication de ces divers noms, pôles et qualificatifs permet de mettre au jour des dispositions intellectuelles rémanentes, réajustées au fil des investissements successifs de Schaeffner, que nous retrouverons dans les écrits ethnologiques ultérieurs ${ }^{9}$.

\section{La formation d'un goût puriste}

Schaeffner est issu de la bourgeoisie parisienne cultivée. La plupart des hommes de la famille paternelle (père, oncles et plusieurs cousins) sont chimistes, en Allemagne, aux États-Unis ou en France. Son père, chimiste indépendant (ni salarié, ni universitaire), quitte l'Alsace en 1870 et épouse une femme de la bourgeoisie parisienne, qui tient salon à leur domicile du 5 rue de Creveaux ( $\mathrm{XVI}^{\mathrm{e}}$ arrondissement). Ils ont un fils unique, André, le 7 février 1895, qui se destine à la chimie de façon «naturelle ». Toutefois, sa mère lui enseigne solfège et piano dès l'âge de quatre ans, et le place ensuite auprès de professeurs privés - les deux parents chantent, la mère est pianiste amatrice. C'est au cours d'un séjour

\footnotetext{
${ }^{7}$ Musicographie désigne conjointement la critique musicale et la musicologie, ces deux activités étant alors faiblement différenciées - et Schaeffner appartenant, comme nous le verrons, à la génération qui contribue à instituer une musicologie « scientifique».

${ }^{8}$ SERRY, 2004.

9 Sur le champ musical dans l'entre-deux-guerres, $c f$. notamment CHEYRONNAUD, 1991 ; DUCHESNEAU, 1997 ; FAURE, 1997 ; FUlChER, 1999a ; MAISONNEUVE, 2003 ; CHIMÈNES, 2004.
} 
estival à Cologne, chez un ami paternel, précédant son entrée au lycée (1910), que se cristallisent ses premiers goûts personnels - il indiquera que ses parents chantaient «très bien » mais «malheureusement pas toujours de la bonne musique ${ }^{10}$. Il découvre en effet, grâce à des revues de son hôte, l'impressionnisme pictural et l'architecture moderne ${ }^{11}$. À son retour, il lit Maeterlinck et les symbolistes, puis les écrivains catholiques liés à la toute jeune Nouvelle Revue Française (Paul Claudel, Maurice Barrès, Charles Péguy, André Gide, Marcel Proust) ${ }^{12}$. Ces premières préférences à la fois puristes et spiritualistes se voient développées par la fréquentation des «réunions d'Eylau». Il s'agit de séances dominicales organisées par l'abbé Cornette pour les élèves du lycée Jeanson-de-Sailly, qui substituent au catéchisme traditionnel une pédagogie lettrée et «moderne ${ }^{13}$. Il est orienté aussi, en musique, par ses professeurs de piano, qui le confrontent à Debussy, Strawinsky, Albeniz et Granados, soit les compositeurs modernistes que se disputent ou s'opposent les deux sociétés de concert puristes rivales - la Société Nationale de Musique (SNM), créée en 1871 et liée à la Schola Cantorum (créée contre le Conservatoire en 1894) et à la figure de Vincent d'Indy (soit le pôle scholiste ou d'indyste); et sa scission, la Société de Musique Indépendante (SMI) créée en 1909 par Gabriel Fauré (directeur du Conservatoire depuis 1905) et Maurice Ravel. Ces deux identifications esthétiques se rejoignent dans la lecture des critiques littéraires et musicales de Jacques Rivière, secrétaire de rédaction de la $N R F$ de janvier 1912 à août 1914 sous la direction de Jacques Copeau ${ }^{14}$. Elles se cristallisent avec le « choc » du Sacre du printemps, auquel il assiste un an après sa création et les polémiques qu'il a suscitées, à l'occasion de sa reprise au Casino de Paris (mai 1914) ${ }^{15}$.

À la sortie du lycée en 1914, Schaeffner évite l'incorporation militaire afin de préparer le concours de l'Institut de chimie appliquée, auquel il échoue l'année suivante. D'après Denise Paulme (sa future femme), il s'agit d'une sorte d'acte manqué - «son choix était déjà fait », celui de la musique ${ }^{16}$. Mobilisé comme bibliothécaire au Musée de la guerre, s'ouvre alors une période d'expériences lettrées multiples : concerts, théatre et salons ${ }^{17}$; cursus de l'École du Louvre (alors souvent fréquentée moins pour acquérir des compétences professionnelles que pour parfaire une « culture»), avec notamment les cours de l'archéologue et historien des religions Salomon Reinach; fréquentation assidue des réunions dominicales des frères Baruzi, dont l'aîné Joseph travaille aussi au Musée de la

${ }^{10}$ DuVElle, $1982 \mathrm{~b}, 369$.

${ }^{11}$ Ibid.

${ }^{12}$ PAULME-SCHAEFFNER, 1982a, 362.

${ }^{13} \mathrm{La}$ « réunion d'Eylau » est créée en 1897 par l'abbé Cornette, le lycée ne disposant pas d'aumônier. Pendant la guerre, il s'associera avec des expérimentations de scoutisme et contribuera à la création en 1920 des Scouts de France. Ce lieu de formation «morale» des enfants de la bourgeoisie parisienne est alors situé au pôle maurrassien, comme l'attestent, explicitement, trois conférences qu'y donnera Schaeffner en 1915 et 1916 : «Wagnerisme et debussysme» (133 pages), «Chopin, Schuman, Lizst» (19 pages), «Sur Sainte-Beuve» (51 pages) - Fonds Schaeffner de la Médiathèque musicale Mahler (désormais indiqué F.S.), Dossiers de travail, 6.

${ }^{14}$ Cf. la compilation de RIVIÈRE, 1911. Sur l'évolution des positions autonomistes « œcuméniques » (de la référence classique à l'intégration des avant-gardes) et des luttes internes de la $N R F$ - et notamment sur ses fractions catholiques, à la fois maintenues et peu à peu excentrées au cours des années 1920 - $c f$. SAPIRO, 1999 ; SERRY, 2004.

${ }^{15}$ PAULME-SCHAEFFNER, 1982a, 362.

${ }^{16}$ PAULME-SCHAEFFNER, 1982a, 362.

${ }^{17}$ Marcel Belvianes, rencontré en 1915 par un ami commun anarchisant, camarade de régiment de Belvianes et ami d'enfance de Schaeffner, se souvient de danses balinaises, de promenades au parc de Versailles, de la Comédie-Française (que Schaeffner découvre), du cercle des frères Baruzi ( $c f$. infra), du salon musical d'une professeure de chant, sœur d'un de leurs amis, «Paul $M »$ (BELVIANES, 1982). 
guerre ( $c f$. infra). Il faut ici relever que Schaeffner, pour expliquer à Charles Duvelles son abandon de la chimie, évoquera les réunions d'Eylau, mais aussi la « chute du père », avec l'ironie ambivalente caractéristique de la menace du déclassement: "Heureusement que mon père était un très mauvais commerçant. Il a fait faillite. Alors il est tombé dans les assurances $\gg{ }^{18}$. L'instabilité de la trajectoire de Schaeffner ${ }^{19}$ semble ainsi favorisée par la rémanence d'un habitus clivé, entre statut social d'origine élevé et incertitude sur son maintien (menace du déclassement, puis positions prometteuses mais risquées, "à faire », et peu lucratives comparées au capital économique parental), entre univers paternel (activité de savoir, d'ailleurs centrée sur la méthode classificatoire, mais indexée à la sphère économique et au modèle de l'entrepreneur) et univers maternel (culturel et lettré, mais hédoniste et non puriste, et plus «élevé » socialement que celui du père) ${ }^{20}$. On peut donc trouver là un facteur de l'indécision et des déplacements positionnels des années 1920, de l'attrait pour des positions à créer (la musicologie savante) et risquées (l'étude « décalée » sur le jazz, l'ethnologie musicale), puis l'investissement d'un double statut d'ethnologue et de musicologue.

La fréquentation des cours de Reinach et surtout du cercle Baruzi déterminent en grande partie les orientations ultérieures de Schaeffner. Salomon Reinach (1858-1932), conservateur adjoint du Musée des antiquités nationales de 1893 à 1902, puis son directeur jusqu'en 1932 (le durkheimien Henri Hubert le remplaçant comme conservateur adjoint), promeut une archéologie scientifique fondée sur la collecte et la classification systématique d'objets, et sur les méthodes philologiques appliquées aux textes anciens - par opposition à l'érudition lettrée des collectionneurs et archéologues amateurs, ou aux usages politiques des savoirs historiens sur l'Antiquité. Par ses cours, Schaeffner découvre donc moins une discipline constituée qu'une pratique de savoir «muséale » ou «muséocentrée » exhibant ses méthodes et son idéal de vérité, qu'il associera à l'organologie et retrouvera dans l'ethnologie. La mesure de cette découverte reste toutefois malaisée, l'approche « laïque » des religions que promeut Reinach étant alors mal perçue par les intellectuels catholiques. En sens inverse, le pôle Baruzi ( $c f$. infra) se distingue, au sein de la nébuleuse catholique, par son caractère universitaire, c'est-à-dire savant plutôt que prophète ou expert ${ }^{21}$, possible point d'accroche avec les pratiques d'un Reinach - auquel cas la proximité relative d'Henri Hubert avec les milieux catholiques pourrait y avoir contribué.

Quant aux frères Baruzi, c'est un ensemble d'intérêts, de questionnements et de schèmes intellectuels plus larges ou «englobants » qu'ils procurent à Schaeffner - mais

\footnotetext{
${ }^{18}$ DUVELLE, 1982b, 369, nous soulignons.

${ }^{19}$ Instabilité en termes d'identifications et de positions, qui se traduit aussi en termes de statuts et de revenus. Dénué de diplôme universitaire, il est, en ce qui concerne ses activités rémunératrices, membre des rédactions du Ménestrel et de La Revue Musicale de 1921 à 1929, secrétaire artistique de l'Orchestre symphonique de Paris de 1928 à 1931, assistant-provisoire au Museum national d'histoire naturel en 1928, boursier de la Caisse nationale des sciences en 1934, chargé de recherche à la Caisse nationale de la recherche scientifique en 1937, maître de recherche au CNRS en 1941, sans jamais atteindre le statut de directeur de recherche, ne pouvant ainsi diriger des thèses - à l'inverse de Michel Leiris (en 1967); Marcel Griaule devient quant à lui professeur à La Sorbonne dès 1943, Denise Paulme-Schaeffner directrice d'études à la VIe section de l'EPHE en 1957. Il est refusé au poste de sous-directeur du Musée de l'homme en 1938 (quand Georges Henri Rivière, prenant la direction des ATP, le laisse vacant), et en 1947 (quand Paul Rivet en reprend la direction). De même, ses revenus ont toujours été relativement limités, et au moins à partir des années 1950, inférieurs à ceux de sa femme. Après sa retraite en 1967, il s'est «retiré du monde» avec semble-t-il une certaine amertume. Nous devons ces informations à Jean Jamin.

${ }^{20}$ Bien qu'ayant mené une (double) carrière de savant, Schaeffner se définit continûment comme un esthète bohème - ainsi Denise Paulme-Schaeffner relève : " "Savant peut-être, artiste d'abord". Tel, en pleine lucidité, André Schaeffner se définissait quelques jours avant sa mort » (PAULME-SCHAEFFNER, 1982a, 362).

${ }^{21}$ Sur la distinction et les rapports entre ces trois modèles d'intellectuel, $c f$. FASSIN, 1998.
} 
aussi un réseau d'affinités relativement dense, identifié d'un côté à la figure d'Henri Bergson, et de l'autre, au pôle catholique de la $N R F^{22}$. C'est notamment à travers eux que Schaeffner lit et est durablement marqué par l'approche généalogique et anti-rationaliste de Nietzsche, et surtout par l'intuitionnisme de Bergson. En effet, Jean Baruzi (1881-1953) est un philosophe catholique réputé - il reprendra en 1926 la chaire d'histoire des religions occupée par Alfred Loisy au Collège de France, où il se rapprochera (suivi par Schaeffner) de Gabriel Marcel (1889-1973) ${ }^{23}$. Sa thèse consacrée à Saint Jean de la Croix et le problème de l'expérience mystique connaîtra un certain succès en 1924, car il y professe un anti-rationalisme catholique non dogmatique, réhabilitant par exemple les théologies protestantes, et se définissant comme un exégète savant et non théologien ${ }^{24}$. Quant à Joseph Baruzi, l'aîné, il a publié Le Rêve d'un siècle, Victor Hugo et Richard Wagner en 1904 (dédié à Bergson), puis La Volonté de métamorphose en 1912 (dédié à Charles du Bos, membre de la $N R F$ ), où il développe de même une métaphysique spiritualiste catholique appuyée sur l'intuitionnisme bergsonien ${ }^{25}$, durablement endossée par Schaeffner. Il est aussi critique au Ménestrel, revue musicale installée (la plus ancienne), où il fera entrer ce dernier ( $c f$. infra). Celui-ci le considère ainsi comme son «père spirituel», comme le rappelle Denise Paulme dans la plupart de ses notices biographiques ${ }^{26}$.

\section{Du scholisme au modernisme}

L'inscription de Schaeffner, après la guerre, aux cours de composition de Vincent d'Indy à la Schola Cantorum marque son renoncement à la carrière de pianiste. S'il s'attachera à minimiser cette expérience plus tard ${ }^{27}$, ce choix paraît «naturel » au sortir de la guerre : l'école et ses ramifications (éditions, concerts, réseau de musicographes) se définissent à la fois par leur entreprise d'autonomisation du champ musical et par leur spiritualisme catholique ; elle est dirigée par Vincent d'Indy, principale figure du champ

${ }^{22}$ Dans un hommage à Joseph Baruzi, Édouard Dolleans (proche des historiens communistes) énumère les liens des frères Baruzi pour la période ultérieure : Union pour la Vérité, rencontres de Pontigny, Paul Desjardins, Jean Schlumberger, Jean Paulhan, Gabriel Marcel, Marcel Arland (DoLLEANS, 1954).

${ }^{23}$ Pour situer ce dernier dans la «nébuleuse catholique (l'un des premiers importateurs de la phénoménologie allemande et parfois considéré comme l'«inventeur » de l'existentialisme), $c f$. SAPIRO, 1999 ; SERRY, 2004.

${ }^{24}$ BARUZI JEAN, 1911.

${ }^{25}$ BARUZI JOSEPH, 1904 et 1911.

${ }^{26}$ Par exemple : «Il tenait (Joseph Baruzi) plus ou moins pour son père spirituel » (PAULME-SCHAEFFNER, in SCHAEFFNER, 1998a, 7). Schaeffner lui dédie en 1957 son édition des lettres de Nietzsche à Peter Gast (NIETZSCHE, 1957) - le premier volume est exclusivement composé des notes et de la longue introduction, 209 pages centrées sur les thèses concernant les origines musicales du drame - le «masque » (au sens de jeu, travestissement de la «nature animale » de l'homme) est le premier geste qui fonde la musique ; le drame (grec) en hérite antérieurement à tout investissement du $\log o s$ : thèse interprétée comme une conception anti-rationaliste des fondements de l'humanité. Ces thèmes structurent les écrits de Schaeffner, notamment l'Origine des instruments de musique (cf. infra).

${ }^{27}$ «Je n'aimais pas son école (la Schola Cantorum de d'Indy), mais lui était un excellent musicien, excellent instrumentiste » (DUVELlE, 1982b, 368). Dès 1927, Schaeffner rendra compte de La Schola Cantorum, son histoire depuis sa fondation jusqu'en 1925 en soulignant la réhabilitation «puriste » des musiques anciennes (musiques grégoriennes, palestriniennes, Monteverdi, maîtres français anciens, Bach, Haendel, polyphonies médiévales...), et son influence sur les jeunes compositeurs, mais en citant les moins «scholistes » (Debussy, Ravel, Milhaud, Satie), et en pointant l'appropriation de redécouvertes qui auraient en réalité été faites par d'autres, le «mépris pour certains jeunes musiciens contemporains », et l'orientation politique de d'Indy (SCHAEFFNER, 1927d, 181). 
musical depuis le début du siècle ${ }^{28}$. Par ailleurs, cette expérience est déterminante à plus d'un titre.

Premièrement, il accumule un capital social nouveau qui lui permet notamment d'intégrer l'équipe de la toute jeune Revue Musicale. Grâce à Joseph Baruzi, il avait commencé à publier des comptes rendus de concerts dans Le Ménestrel ${ }^{29}$. Mais ses trois premiers articles de fond sont publiés en 1922 et 1923, pour l'un, dans Le Ménestrel ${ }^{30}$, et pour les deux autres, dans le cadre de dossiers confiés par La Revue Musicale à des figures scholistes - et Schaeffner devient peu après membre de l'équipe de la revue ${ }^{31}$. De plus, ils traitent de thèmes parmi les plus classants : Wagner et César Franck, soit les deux figures tutélaires du pôle scholiste. Cependant, ils font ressortir l'orientation moderniste et sensualiste de Schaeffner, autrement dit une variante formaliste des thèses dominantes sur l'histoire de la musique initialement élaborées dans le cadre spiritualiste ou moraliste imposé par d'Indy et ses alliés ${ }^{32}$. Dans son article sur César Franck, Schaeffner relève ainsi les innovations du maître, mais pour faire porter l'ombre de la rupture scholiste jusqu'à des compositeurs «aussi différents » qu'Albéric Magnard ou Albert Roussel, «à qui, soulignet-il, s'est voué l'attachement d'un Darius Milhaud ${ }^{33}$. Or, Albéric Magnard (1865-1914) utilise le purisme moraliste d'un d'Indy pour instancier des positions politiques dreyfusardes et républicaines; Albert Roussel (1869-1937), certes formé à la Schola Cantorum, a rejoint la SMI, fondée contre la SNM, dès sa création ; enfin, Darius Milhaud est alors la principale figure de la jeune avant-garde des «Six », qui expérimente les innovations poly- et a-tonales et les emprunts aux genres vulgaires ${ }^{34}$ - une façon, donc, de

${ }^{28}$ Il est aussi membre fondateur de la Ligue de la patrie française puis lié à l'Action française (une éphémère société de concerts, la Schola Cantorum d'Action Française, est créée en 1920). Cependant, en tant qu'un des principaux acteurs de l'autonomisation du champ (dans sa variante moraliste, «spirituelle », et non formaliste), son prestige musicien déborde ses prises de position politiques: il attire des compositeurs, instrumentistes, musicographes (apprentis et installés) aux orientations musicales et politiques variées, voire opposées.

${ }^{29}$ Le premier date du 4 juin 1920. Il est membre de la rédaction jusqu'au 26 avril 1929 (PAULMESCHAEFFNER, 1982b; KRADER, 1958).

${ }^{30}$ SCHAEFFNER, 1922a.

${ }^{31}$ La Revue Musicale est créée en 1920 (Henry Prunières en est le directeur, André Cœuroy le rédacteur en chef de 1924 à 1937). Ses locaux jouxtent ceux de la NRF, et les échanges de collaborateurs sont fréquents - Boris de Schloezer, arrivé à Paris en 1921 ( $c f$. infra), étant membre permanent des deux équipes. Prunières organise dès 1920 des Concerts de la Revue Musicale (qui font rapidement long feu) dans les locaux voisins du Théâtre du Vieux-Colombier, eux-mêmes directement liés à la $N R F$, par Jacques Copeau et Paul Claudel notamment. La programmation, variée, est cependant un peu plus engagée vers les «modernes » que celles de la SNM ou de la SMI, intégrant notamment l'avant-garde des « Six » (DUCHESNEAU, 1997).

${ }^{32}$ Leur entreprise d'autonomisation de la musique est passée entre autres par le développement de la musicographie sous ses modalités historiennes et folkloristes, comme restitution d'un passé pré-moderne et de ses survivances : «La régénération musicale à laquelle entendent se consacrer les fondateurs de la Schola dépendra pour beaucoup de cette restauration musicale catholique d'obédience modale, son apologétique du chant grégorien - le modèle solesmien, bien sûr - et dans le sillage, la chanson populaire. En somme, un "retour à": retour aux premiers aliments du goût musical, à la cantilène monodique d'avant l'avènement de l'individualisme et les grandes ruptures religieuses (la Réforme et ses "conséquences" : Révolution, romantisme), âge d'or d'une société "intégralement" chrétienne (écho du "nationalisme intégral" de Maurras), de l'unisson des exécutions en commun lui-même symbole d'un "lien social" d'une solidité éprouvée entre dignitaires et plus humbles ». Cette réélaboration de l'histoire musicale européenne, qui cherche avant «la grande rupture monteverdienne (...) la coupure irréparable entre le vieux système de plain-chant (l'antique tonalité) (...) et celui de notre tonalité moderne », présente une continuité depuis Joseph d'Ortigues et François-Joseph Fétis (1784-1871) jusqu'aux musicographes scholistes tels que Julien Tiersot ou Lionel de la Laurencie ( $c f$. infra) qui fonderont en 1917 la Société française de musicologie (CHEYRONNAUD, 2003, $193 ; 50$ ).

33 SCHAEFFNER, 1922b, 154. Le numéro comprend aussi Julien Tiersot, Henry Duparc (deux figures d'indystes), et Boris de Schloezer.

${ }^{34}$ FULCHER, 1999b ; FAURE, 1997. 
signifier l'autonomie des innovations formelles par rapport à leurs usages politiques, proche des positions « œcuméniques » de la $N R F$. Quant aux rapports de Wagner à l'opéra français (genre ennemi pour les scholistes), Schaeffner les explique par des raisons ambiguës en regard des thèses d'indystes ${ }^{35}$. Wagner voyait selon lui l'opéra français comme un «vaudeville agrandi», proche de la «vie populaire parisienne» révélée par les trois révolutions, où la musique restait heureusement indexée à la danse et à la chanson. Or, cette proposition renvoie certes à la nostalgie « scholiste » d'une fusion des différentes pratiques musicales au service de l'union spirituelle de l'organisme social, que la modernité aurait dissociées, mais pour l'orienter vers des signifiés opposés : le vaudeville moderne et vulgaire n'est pas l'«antique chanson populaire» des folkloristes, et les révolutions auraient désagrégé, et non révélé, la «vie populaire » que visent les d'indystes. Enfin, la conclusion de l'article explicite la variante sensualiste, et non spiritualiste, de son antirationalisme : «à côté des motifs d'ordre plutôt théorique (rationnel et moral) que Wagner nous donne d'avoir cédé aux influences françaises, il nous faut rechercher les raisons plus matérielles, plus sensorielles qui sourdement ont agi sur ses sympathies artistiques ${ }^{36}$.

Ce sensualisme renvoit ainsi à la seconde expérience déterminante du passage de Schaeffner par la Schola Cantorum. À son entrée, d'Indy décèle des faiblesses rythmiques et lui conseille de suivre les cours de «solfège rythmique »d'Albert Jeanneret : «ça a été mon départ décisif. Alors, j'ai fait énormément de rythmique, aussi bien gymnastique que solfège ${ }^{37}$. C'est qu'il découvre, avec la gymnastique rythmique, les conceptions philosophico-musicales élaborées par Émile Jaques-Dalcroze (1865-1950). Le schème central consiste à faire du rythme (la pulsation « corporelle» que la mesure, convention graphique qui ponctue régulièrement les portées des partitions, vient cadrer «rationnellement»), tel qu'opposé à l'harmonie (avec la mélodie comme entité «émotive », intermédiaire entre le rythme corporel et l'harmonie rationnelle), la base de toute pédagogie musicale, mais aussi de tout épanouissement personnel. Alors que le solfège dominant envisage la musique comme une langue, dont il faut en premier lieu apprendre l'alphabet (les notes) puis les règles d'association (gammes, modes, accords, rythmes... jusqu'au «plus complexe»: chiffrages, contrepoint...), Jaques-Dalcroze préconise de commencer avec des exercices gymniques qui associent un mouvement corporel à un mouvement sonore (décomposant ainsi non seulement des notes, mais des rythmes, des intensités, des timbres...) - d'apprendre le «sens musical» avant «la musique ».

Ainsi, avec les cours d'Albert Jeanneret, Schaeffner non seulement développe une compétence rythmique, mais investit une pratique liée aux milieux lettrés parisiens, et un corpus théorique associé plus ou moins directement au bergsonisme et aux divers antirationalismes ${ }^{38}$. Et c'est bien ce flou qui explique à la fois qu'il endosse ces dispositions au sein de la Schola Cantorum, et que celles-ci contribuent à l'en éloigner tout en maintenant un sentiment de continuité : alors que certains insistent sur la critique du logocentrisme et le passage par le corps pour accéder aux réalités spirituelles sans la médiation «asséchante » du langage rationnel, Schaeffner fait partie de ceux qui «redécouvrent» avec la

${ }^{35}$ SCHAEFFNER, 1923b. Le numéro, introduit par Paul Dukas, comprend entre autres André Suarès, JacquesGabriel Prod'Homme (secrétaire de la Société française de musicologie, $c f$. infra) ou André Georges, liés au pôle d'indyste.

${ }^{36}$ Ibid., 125, il souligne.

${ }^{37}$ Duvelle, $1982 \mathrm{~b}, 368$.

${ }^{38}$ Pour se faire une idée du rôle joué, dans les arts de la scène (notamment autour du Théâtre du VieuxColombier et de la $N R F$, et des Ballets russes et suédois), par Jaques-Dalcroze et son associé à l'Institut d'Hellereau qu'il fonda en 1911, le scénographe Adople Appia, on consultera la biographie de BERCHTOLD, 2000, ainsi que LAMBERT, 1998. 
gymnastique rythmique le «fonds corporel» de l'être humain, pour le tirer vers une sorte de vitalisme esthétique opposé à «l'abstraction» des arts et des théories « rationalistes », « coupés de la vie réelle ». Ses comptes rendus des méthodes publiées par Jaques-Dalcroze et Jeanneret pointent ainsi un schème cognitif rémanent, celui de l'opposition entre le rythme corporel, vital, et la mesure abstraite, morte, imposée par le cadrage graphique, rationnel, de la musique écrite :

«Qui a en effet pratiqué la méthode Dalcroze, sous forme soit de solfège, soit de gymnastique, sait quel définitif apport dans l'enseignement de la musique aura été cette distinction à jamais éclaircie de la mesure et du rythme, à jamais imprimée corporellement, au point même que la chorégraphie de Noces (Strawinsky et Ballets russes) semble l'exsuder par tous ses gestes $\gg{ }^{39}$.

C'est en vertu des mêmes dispositions « rythmiques » que Schaeffner rencontre Wanda Landowska, interprète réputée qui tente de réhabiliter le clavecin et les conventions d'interprétation de Bach et de ses contemporains - et qu'il rencontre aussi les musicographes qui, autour de Lionel de La Laurencie, s'inscrivent dans le même mouvement de promotion et de documentation de cette musique dès lors dite « ancienne » (aujourd'hui appelée baroque ${ }^{40}$ ). Il écrit un compte rendu élogieux de l'une de ses prestations semble-t-il en $1923^{41}$, et celle-ci l'invite dès lors à son salon qu'elle tient à Saint-Leu (où elle fondera en 1925 une École de musique ancienne avec Alfred Cortot). Là, il rencontre Marc Pincherle, jeune musicologue «savant» de la génération de Schaeffner, avec qui il collaborera, et Ernest Ansermet, jeune compositeur, chef d'orchestre et critique suisse qu'il retrouvera au sein de l'Orchestre symphonique de Paris ${ }^{42}$. C'est probablement aussi par ce biais qu'il est sollicité par Bertrand Guégan afin d'éditer les poèmes de Ronsard mis en musique par les compositeurs de son temps ${ }^{43}$.

Cette publication sera évoquée par Schaeffner comme le point de rupture avec ses années de jeunesse, en la situant de façon assez énigmatique à l'origine de son arrivée ultérieure au Musée de l'homme :

«Mon point de départ, c'est un type que vous n'avez jamais connu, qui s'appelait Bertrand Guégan. C'est un excellent historien de la cuisine française, grand ami de... d'un tas de gens. (...) Il connaissait très bien Ravel. (...) C'est par Ronsard que j'ai été amené à le connaître. J'ai fait un livre avec Bertrand Guégan sur Ronsard. Et c'est comme ça que j'ai connu Payot $\gg{ }^{44}$.

Ravel est l'une des principales figures démarquées des «d'indystes », même s'il ne fait pas école. Parmi le «tas de gens » rencontré, se trouvent probablement les réseaux autour de Jean Cocteau et Blaise Cendrars (dont l'Anthologie nègre ${ }^{45}$ a servi de base au livret de La Création du Monde de Darius Milhaud, en 1922), c'est-à-dire la jeune avant-garde musicale : autant bibliophile qu'historien, Guégan a publié entre 1920 et 1922 un Almanach de Cocagne aux éditions de la Sirène que ceux-ci ont créées en $1918^{46}$. Enfin, les frères Payot, éditeurs lausannois, ont ouvert un bureau parisien avant la guerre, et investi

\footnotetext{
${ }^{39}$ SCHAEFFNER, 1926a.

${ }^{40}$ Sur le « renouveau baroque » et ses liens avec les débats pédagogiques autour de Dalcroze, $c f$. HENNION, 1993.

${ }^{41}$ Il concluera un article-hommage à Landowska par cette mention : «juillet 23-mai 27 », la première date renvoyant probablement à leur rencontre (SCHAEFFNER, 1927b).

${ }^{42}$ Il fut notamment le chef d'orchestre des spectacles des Ballets Russes (qui réalisèrent le Sacre du printemps en 1913) de 1915 à 1923.

${ }^{43}$ RONSARD, 1924.

${ }^{44}$ DUVELLE, $1982 \mathrm{~b}, 367$.

${ }^{45}$ CENDRARS, 1921.

${ }^{46}$ GuÉGAN, 1920-1922.
} 
notamment le domaine des essais para-universitaires et des sciences humaines nouvelles et « en vogue ». Et développant leur catalogue universitaire, ils publieront les deux ouvrages grâce auxquels Schaeffner se fera un nom : la révision du Dictionnaire d'Hugo Riemann en 1931, l'Origine des instruments de musique en 1936 (cf. infra).

Cependant, il faut ajouter que cette publication lui vaut aussi deux articles remarqués ${ }^{47}$, et le parrainage du musicographe «savant» Lionel de la Laurencie. En effet, celui-ci a repris la présidence de la Société française de musicologie, qu'il a contribué à fonder en 1917, en janvier $1924^{48}$; et Schaeffner s'y voit accepté comme membre un mois plus $\operatorname{tard}^{49}$. De plus, c'est à ce moment que La Laurencie le sollicite pour deux articles de l'Encyclopédie de la musique et Dictionnaire du Conservatoire ${ }^{50}$ : le premier, co-écrit avec Boris de Schloezer, paraît en 1925 ; le second, étude d'organologie sur le clavecin, paraît en $1927^{51}$. Or, on peut interpréter ces deux textes comme des thématisations, dans le registre savant, des déplacements produits par les diverses socialisations ultérieures à l'entrée de Schaeffner à la Schola Cantorum. Celui consacré au clavecin s'appuie sur la documentation réunie par Wanda Landowska, dont il reprend les conceptions (et à qui il dédie le texte). Il y dénonce en introduction la domination du piano et de la tonalité tempérée à partir du $\mathrm{XIX}^{\mathrm{e}}$ siècle, qui aurait disqualifié la musique ancienne et ses instruments. Et conclut, après une étude minutieuse de l'histoire du clavecin et de ses usages (matériaux, factures, qualités acoustiques, solutions techniques aux insatisfactions liées à l'évolution des conventions...), sur l'importance des questions d'interprétation - «l'art de phraser » selon Landowska, où l'instrumentiste compte autant que le compositeur, et où, dans la continuité du vitalisme dalcrozien, la sensibilité s'oppose à la rationalité ${ }^{52}$.

L'article co-écrit avec Boris de Schloezer marque, quant à lui, l'adoption définitive par Schaeffner d'une position formaliste, et l'éloignement conséquent du pôle scholiste. Schloezer (1881-1969) s'est installé à Paris en 1921, fuyant le pouvoir soviétique. Il entre rapidement à La Revue Musicale et à la $N R F$, où il devient le critique musical et le principal artisan de la découverte du roman russe (il traduit la plupart des romans, et dirige la collection «Jeunes Russes » de la NRF à partir de 1926). Il adopte ainsi les positions de la $N R F$ : autonomie des arts face à la morale et la politique, et intérêt conséquent tant pour le classicisme que pour les recherches formelles des avant-gardes - ce qui ne l'empêche pas d'avoir une conception «spirituelle» ou «idéaliste» des arts. Beau-frère

\footnotetext{
${ }^{47}$ SCHAEFFNER A., 1924a ; $1924 \mathrm{~b}$.
}

${ }^{48}$ La Laurencie fait partie des réseaux scholistes, et alterne à la présidence de la SFM avec Julien Tiersot, lui aussi scholiste, durant toutes les années 1920 - le premier en 1917-1920, 1924-1927, 1932-1933; le second en 1921-1923 et 1927. Tous deux promeuvent une musicologie érudite et savante, essentiellement fondée sur le modèle philologique - $c f$. les articles de Rémy Campos et Sophie-Anne Leterrier dans ce numéro.

49 SCHAEFFNER, 1967. Il a été approché par Julien Tiersot dans le cadre d'une campagne d'adhésion, et parrainé par Tiersot et La Laurencie (lettre de Julien Tiersot à André Schaeffner, 10 janvier 1924, FS, Dossiers de travail, 10, « Musicologie »).

${ }^{50}$ Cette entreprise éditoriale participe de la fondation d'une musicologie « savante » démarquée de la critique journalistique - par l'intérêt porté à l'histoire de la musique plutôt qu'à l'actualité, plus que par l'élaboration de méthodes et de questionnements spécifiques (cf. l'« Avis aux lecteurs » du 1er Bulletin de la SFM, en mars 1917, qui parle de «science nouvelle », d'archives, de bibliothèques et de contribution à la «science française », pour «faire revivre le passé musical de notre pays»); d'ailleurs, les membres de la SFM sont le plus souvent aussi, voire d'abord journalistes. Elle a été lancée en 1913 par Albert Lavignac, alors directeur du Conservatoire, puis reprise à sa mort par La Laurencie, à un moment où la domination scholiste conduit à une tentative, éphémère, de rapprochement du pôle Conservatoire-SMI (DUSCHENEAU, 1996).

${ }^{51}$ SCHAEFFNER, SCHLOEZER, 1925 ; SCHAEFFNER, 1927 a.

${ }^{52}$ Cf. aussi SCHAEFFNER, 1927b : hommage à Landowska et à son entreprise de réhabilitation de la musique ancienne et de l'interprète, en alliance avec les musicographes «savants » Romain Rolland, La Laurencie, André Pirro et Henri Quittard. 
d'Alexandre Scriabine dont il promeut le statut de précurseur quant aux ruptures avec le système tonal, il importe aussi un regard un peu plus bienveillant sur l'avant-garde viennoise que la plupart des critiques et compositeurs français - l'un des rares soutiens d'Arnold Schönberg à Paris, dès 1921, avec Arthur Hoérée ou Maurice Ravel ${ }^{53}$. L'identification de Schaeffner à ce type de position, notamment après l'échec de son livre sur le jazz co-écrit avec le rédacteur en chef de la Revue Musicale ( $c f$. infra), se repère explicitement dans le jeu des citations croisées et des comptes rendus réciproques. De même, Schaeffner et Schloezer publient les deux premières monographies consacrées à Strawinsky, le désignant tous deux comme l'inventeur de la modernité musicale par la conjonction de l'orientalisme venu de Russie, de Wagner et de Debussy - mais dans des registres différents, plus «spiritualiste» pour Schloezer (qui termine sur le tournant catholique (orthodoxe) de Strawinsky, concomitant de sa radicalisation politique à droite), plus «savant» pour Schaeffner (qui décompose minutieusement les sources de l'œuvre, en s'appuyant sur les entretiens qu'il a eus avec l'auteur en 1929) ${ }^{54}$.

\section{II - L'exotisme comme extension critique du domaine musicologique}

La distanciation relative d'avec le pôle d'indyste par lequel Schaeffner est entré en musicographie s'achève ainsi, au terme de diverses expériences socialisatrices, par l'investissement, à partir de 1924 environ, d'une position «savante » (avec la SFM) et «autonomiste» (avec La Revue Musicale) qui participe de l'imposition de l'humeur «néoclassique » en laquelle l'historiographie ultérieure résumera la période. Schaeffner suit en cela l'évolution de La Revue Musicale, rapidement consacrée au pôle puriste du champ musical, et où il représente, avec Schloezer notamment, la fraction moderniste. Cependant, Schaeffner développera, par la suite, ses investissements savants et académiques, que ce soit en musicologie ou en ethnologie, au détriment de ses activités critiques, dont on peut supposer qu'elles ne satisfont pas les ambitions d'un jeune lettré relativement déclassé. En effet, la faible réception de son premier ouvrage, Le Jazz (1926), anticipé comme un «coup » et conçu comme une prise de position personnelle et inédite, viendra en quelque sorte révéler à ses yeux cette position incertaine et le décalage relatif de ses dispositions avec les possibles qu'elle ouvre. L'intérêt suscité par l'analyse des origines « africaines » du jazz auprès de lettrés situés entre surréalisme et ethnographie, lui fournit alors une sorte de ligne de fuite, à la fois acceptable et ambivalente, du côté de l'exotisme et de ses thématisations « anti-occidentales ». De plus, ses dispositions érudites, ancrées dans la reconversion des premiers investissements lettrés vers l'histoire de la musique (via le cercle Baruzi, l'École du Louvre, le passage par les réseaux scholistes qui l'ont valorisée), se sont trouvées développées avec l'intérêt pour la musique ancienne dans le sillage de La Laurencie, sur un même fond anti-rationaliste.

${ }^{53}$ Leur soutien est embarrassé par la radicalité formaliste de l'atonalisme, qui leur fournit un marqueur d'avant-gardisme tout en les «débordant». Ils opposent ainsi l'abstraction rationaliste de l'atonalisme «allemand », à la vitalité rythmique et/ou à l'émotion mélodique des productions «néoclassiques » «françaises ». Cf., par exemple, SCHLOEZER, 1922 ; HoÉRÉE, 1926. Pierrot lunaire, créé à Berlin en 1902 (la même année que Pelléas et Mélisande de Claude Debussy - avec le Sacre du printemps, ce sont les trois œuvres-phares de la musique moderne), a été représenté pour la première fois à Paris aux concerts Jean Wiéner en 1922, c'est-à-dire par l'avant-garde des «Six »; en 1927, Ravel invite Schönberg à présenter d'autres œuvres à Paris. Schloezer et Schaeffner seront parmi les premiers critiques à soutenir l'avant-garde « sérielle » qui systématisera l'atonalisme après la Seconde Guerre mondiale, autour de Pierre Boulez ( $c f$. l'article de DONIN N. et KECK F. dans ce numéro).

${ }^{54}$ SCHLOEZER, 1929 ; SCHAEFFNER, 1931a. 
Dans cette perspective, l'investissement de l'ethnologie, à partir de 1928, constituera moins une reconversion qu'une extension de l'activité musicographique, dans la continuité directe des premiers folkloristes (Louis Bourgault-Ducoudray, Julien Tiersot, Pierre Aubry). Le titre comme les références et l'aire comparative de la somme de 1936, Origine des instruments de musique. Introduction ethnologique à l'histoire de la musique instrumentale, l'attestent : le détour par les «formes élémentaires » de la musique sert une théorie de « la musique » dans sa plus grande généralité (y compris la musique occidentale savante la plus contemporaine), et une critique des présupposés de la musicologie bien plus qu'une contribution à l'ethnologie (les ethnologues n'y sont d'ailleurs appelés que comme sources). L'ethnologie apparaît à la fois comme une opportunité professionnelle, position « à faire » mais prometteuse en termes de statut (académique) et d'autorité intellectuelle (la critique musicale en quelque sorte sublimée par le registre scientifique), et comme une formation de compromis qui permet de tenir ensemble des aspirations et des dispositions divergentes - entre relativisme ethnologique (et éthique) et essentialisme musicologique, entre croyances scientifiques et croyances anti-rationalistes.

\section{Le jazz comme pivot}

Il faut donc s'arrêter d'abord sur le premier livre publié, en 1926, par Schaeffner, car il apparaît comme pivot à plusieurs titres. D'une part, le livre comme les publications qui le précèdent et l'entourent sont réalisés en collaboration avec le rédacteur en chef de La Revue Musicale, André Cœuroy ${ }^{55}$. Il marque ainsi l'identification au modernisme de La Revue Musicale : ce sont les avant-gardes et les «néoclassiques » qui s'intéressent alors aux jazzbands, intérêt que les «d'indystes » condamnent sèchement. D'autre part, il vient condenser les dispositions intellectuelles engrangées auparavant avec les expériences qu'on dira pour simplifier «catholiques », « archéologiques », «gymniques » et «baroques », pour les élaborer en thèses musicales. C'est cette cristallisation qui explique que le jazz occupera un statut particulier dans les écrits ultérieurs de Schaeffner, venant en quelque sorte signifier par déplacement et condensation ces dispositions forgées antérieurement, depuis l'Origine... jusqu'à ses dernières publications, et ce dans une indépendance relative avec les évolutions du genre musical et de ses définitions - résurgence du primitif dans la musique moderne, il viendrait rappeler cette dernière au rythme, au corps, à la performance qu'elle a occultés ${ }^{56}$. Enfin, cette publication a un double effet en termes d'espace des investissements possibles pour Schaeffner, qui tient au fait que celui-ci y prend au pied de la lettre un «primitivisme nègre » qui n'est bien souvent que rhétorique ou anecdotique chez les critiques et les compositeurs qui s'y intéressent: il produit une étude sérieuse (jusqu'à entendre dans le jazz les origines de la musique) sur un sujet qui est en vogue

${ }^{55}$ Pseudonyme d'André Belime (1891-1976), élève à l’École normale supérieure (section philologie), agrégé d'allemand, il publie, outre des critiques, des études sur les rapports entre musique et littérature - il sera aussi l'une des plumes de la presse vichyssoise, l'une des raisons pour lesquelles Schaeffner reniera leur livre commun.

${ }^{56}$ Nous reprenons ainsi les hypothèses de JAMIN, 1996; JAMIN, WILLIAMS, 2001 : le jazz a eu un «rôle catalyseur dans la formation d'ethnologues de l'entre-deux-guerres » (JAMIN, WILLIAMS, 2001, 18), son centrement sur la performance vive (l'artiste étant l'instrumentiste improvisateur, non le compositeur) fournissant «un modèle intuitif à cette muséographie ethnologique qui se demandait comment procéder pour que les matériaux, "dont la valeur est liée au fait qu'ils sont choses cueillies sur le vif, puissent garder quelque fraîcheur une fois consignés dans des livres ou mis en cage dans des vitrines" » (ibid., 16 - la citation est tirée de LEIRIS, 1938, 344). En effet, le jazz vient cristalliser, du fait de ses propriétés telles que thématisées dans l'entre-deuxguerres, des schèmes intellectuels forgés antérieurement par les socialisations catholiques et musicales : s'il ne les révèle pas (et les produit encore moins), il les active et contribue à leur saisie réflexive et leur élaboration, puis en constitue un signifiant durable. 
précisément parce qu'il n'est pas sérieux. Ainsi, d'un côté, Le Jazz est mal (ou pire : peu) reçu dans le champ musical, et constitue une sorte de «coup manqué », disqualifiant quelque peu ses auteurs (tout au moins Schaeffner, simple critique et signataire des thèses les plus osées, quand Cœuroy est rédacteur en chef de La Revue Musicale et signe les textes les moins compromettants). De l'autre côté, il est à l'origine de la rencontre entre Schaeffner et Georges Henri Rivière, qui résultera dans l'investissement par le premier des activités ethnologiques que le second lui offrira.

Avant d'analyser le cadre intellectuel qui organise l'étude de Schaeffner, il est utile de s'arrêter sur les détails de son élaboration, car ils expliquent en partie l'effet de condensation et d' «emballement» qu'a produit le jazz sur Schaeffner ${ }^{57}$. Au printemps 1925, André Cœuroy et André Schaeffner lancent une « Enquête sur le jazz-band » auprès de personnalités du monde musical, dont le quotidien lettré Paris-Midi publie quarante-neuf réponses. Le jazz-band y est présenté comme faisant partie de la modernité bourgeoise, et suscite des opinions nettement partagées entre les modernistes et les conservateurs. Pour ses défenseurs, dans l'ensemble, il s'agit d'un format orchestral et non d'un genre en tant que tel, identifié à sa culture « américaine » d'origine, à sa mise en avant de la batterie de music-hall et secondairement à l'usage non conventionnel des instruments à vent, et surtout aux rythmes syncopés. Son intérêt résiderait dans les trouvailles d'orchestration, de timbres et de rythmes grâce auxquelles la musique savante serait susceptible de se renouveler - moins par emprunts réels, certains soulignant déjà que ces innovations ont été au moins annoncées, sinon offertes par le Sacre du printemps, que parce qu'il est qualifié au moyen des mêmes catégories valorisées par les modernes: simplicité mélodique, couleurs orchestrales renouvelées par les percussions et les vents, et surtout centralité du « rythme» touchant au corps et au primitif. La question en serait probablement restée là si n'avaient eu lieu, quelques mois plus tard, les polémiques autour de La Revue Nègre (spectacle de music-hall au Théâtre des Champs-Élysées, octobre 1925). Celle-ci résonna avec les aspirations à l'exotisme d'une fraction des jeunes générations lettrées, et fit « découvrir» aux commentateurs musicaux les « origines nègres » du jazz-band ${ }^{58}$. Fortement marqué par ce spectacle, Schaeffner prend au pied de la lettre le schème primitiviste qui a organisé sa réception : il produit une étude minutieuse, exclusivement à partir de récits de voyageurs, de missionnaires et de colons, de « la musique africaine» dans son état primitif sur le sol africain, et modernisé sur le sol américain, pour restituer l'essence «africaine » du jazz, et ce faisant, toucher à l'essence de «la musique» dans sa forme la plus élémentaire. Ces «Notes sur la musique des Afro-Américains » paraissent en feuilleton dans Le Ménestrel au début de l'été $1926^{59}$. André Cœuroy choisit alors de rassembler cette étude (défaite de son appareil de notes) et les réponses à l'enquête de 1925, augmentées d'un chapitre conclusif de sa main qui resitue le jazz-band dans la modernité (en insistant sur ses usages par les compositeurs sérieux, et en valorisant le jazz «blanc» du ragtime et de Paul Whiteman plutôt que le jazz «nègre » sur lequel est centré Schaeffner) ${ }^{60}$ : il s'agit de faire un coup pour lancer la collection « La musique moderne » qu'il vient d'inaugurer chez l'éditeur Claude Aveline (avec une monographie sur Arthur Honegger, par

\footnotetext{
${ }^{57}$ Pour une contextualisation de ces textes (et la reproduction de certains d'entre eux) dans l'espace des discours sur les jazz-bands durant l'entre-deux-guerres, $c f$. MARTIN, ROUEFF, 2002.

${ }^{58}$ Pour une analyse détaillée de cet événement à multiples entrées, $c f$. KLEIN, 1990 ; ROUEFF, 2006. Ce thème apparaissait déjà dans les écrits de musicographes américains publiés en France (d'ailleurs cités dans la bibliographie de Schaeffner): Esther Singleton (1922), Albert Barnes (1924) ; Marion Bauer (1924), Irving Schwerké (1926) - et dans les écrits de Darius Milhaud (par exemple, 1924).

${ }^{59}$ SCHAEFFNER, 1926 b.

${ }^{60}$ Ce chapitre est prépublié dans La Revue Musicale sous le titre «Romantisme du jazz » et signé des deux auteurs (CEUROY, SCHAEFFNER, 1926).
} 
André George ${ }^{61}$ ) - Le Jazz en est le deuxième titre, puis viendront les Essais de Darius Milhaud, Pelléas (de Debussy) de Robert Jardillier, Musique et poésie d'André Suarès, Igor Strawinsky de Boris de Schloezer... Seulement, Schaeffner se souvient qu'il n'eut de retours que d'Albert Schweitzer (l'explorateur), Constantin Brailoiu (musicologue folkloriste, qu'il ne rencontrera qu'au milieu des années 1930), Lionel de La Laurencie et Georges Henri Rivière ${ }^{62}$. Qui plus est, les comptes rendus des critiques alors les plus proches de Schaeffner, Boris de Schloezer et Arthur Hoérée, sont ambigus ${ }^{63}$. Elle place ainsi définitivement Schaeffner du côté des modernes, mais sans produire le «coup » attendu.

Que contient cette étude? Il s'agit d'abord d'élaborer l'autonomie du langage musical par rapport aux significations qu'on peut lui prêter. Et pour cela, de dépasser même la catégorie de musique pure en identifiant l'essence de la musique à ses «formes élémentaires » (titre du premier chapitre : «Musique pure et musique élémentaire »). En effet, la notion de musique pure est encore trop limitative, définissant « un art dont les fins n'excèdent jamais la portée d'un simple problème technique ${ }^{64}$, alors que "la musique glisse hors des cadres qui la voudraient partager en autant d'usages distincts » (9-10) et existe comme une entité spirituelle séparée: "Partout quelque manière d'échapper ainsi au piège d'une explication rationnelle, vers quoi l'idée de musique pure pouvait nous ramener » (12). Or cette musique élémentaire, cette essence de la musique antérieure à la diversité de ses usages (et à toute saisie rationnelle), le jazz la fait toucher du doigt: « $L a$ musique des nègres d'Afrique et d'Amérique, quelle qu'elle soit, ramène directement sous nos yeux le spectacle d'un art proprement élémentaire»(13). C'est que «des musiques violentes d'Afrique aux chants des planteurs des Antilles ou de la Louisiane, aux spirituals, au jazz enfin, il ne s'agit jamais que d'un même fait musical, sous des aspects étrangement peu variés»(14). La suite tente de démontrer cette proposition, par repérage des ressemblances entre les descriptions de «la musique africaine » et de «la musique afroaméricaine », toutes deux différentes dans la variété de leurs formats et la distance temporelle et géographique de leurs diverses manifestations, mais, donc, unifiées comme «musique nègre ». L'explication vient au second chapitre, qui définit ces «AfroAméricains » :

« dans l'exacte mesure où la musique des nègres d'Amérique peut encore recéler quelque chose qui lui vînt de la musique africaine, nous examinerons cette dernière, quitte à en réduire un peu le champ, à ne saisir d'une prodigieuse multiplicité d'instruments que quelques exemplaires, non point peut-être les seuls qui caractérisent le goût musical nègre, mais les seuls dont les planteurs esclaves et leurs descendants aient conservé ou spontanément retrouvé l'usage, et peut-être parce que ces instruments répondaient à l'intime nature de l'esprit nègre $\gg(16)$.

${ }^{61}$ Critique musical aux Nouvelles Littéraires, poste qu'il partage avec Georges Auric, André George fait partie de la nébuleuse catholique maurrassienne - proche par exemple des Cahiers de la Nouvelle Journée, dont il dirige le 5e numéro (1926) - et compte au nombre des amis de Schaeffner - ainsi, Francis Poulenc demande des nouvelles de Georges Auric et de Wanda Landowska, et invite Schaeffner à déjeuner avec «le bon André Georges » (lettre de F. Poulenc à A. Schaeffner, FS, Lettres autographes, octobre 1924, 192); il adresse ses saluts à Roland-Manuel, Georges Auric et André George (ibid., novembre 1925, 197).

${ }^{62}$ SCHAEFFNER, 1980 (1964).

${ }^{63}$ Schloezer souligne l'intérêt de l'étude, mais aussi l'importance de l'improvisation et le rôle du ragtime dans la formation du jazz, c'est-à-dire deux points implicitement opposés à Schaeffner par Cœuroy (SCHLOEZER, 1927). La question du ragtime fait de même le fonds des critiques d'Hoérée - sur le dialogue qui s'engage avec ce dernier, $c f$. MARTIN, ROUEFF, 2002.

${ }^{64}$ Ceeuroy, SchaeffNer, 1988 (1926), 10. Nous indiquons désormais les numéros des pages d'où sont extraites les citations à leur suite, entre parenthèses. 
Une fois posé ce postulat, le raisonnement circulaire peut commencer : il s'agira de chercher les «preuves» de cette «filiation probable» dans «les récits de voyages sur les deux côtes de l'Atlantique » (16).

Logiquement, l'ouvrage va du plus simple au plus complexe, du plus ancien au plus récent : il s'agit de tracer des lignes évolutives. On passe ainsi «Du tambour au balafon » au chapitre 3, qui montre comment «la musique nègre (s'arrache) à l'obsession du bruit », «à cette uniforme matité de bruit qui s'élève de tout le continent noir » (21, 19). Trois chapitres sont ensuite consacrés aux rythmes et percussions, privilège dû à ce que "le tambour enferme ainsi la musique africaine comme sous une chape de plomb et l'oblige à se replier sur elle-même » comme «le nègre, en proie à la jouissance musicale » décrit par Arthur de Gobineau (22). Le principe rythmique qui fait le socle de cette musique nègre tient à la syncopation, « conflit entre rythme et mesure », précise-t-il, en évoquant La Revue Nègre après avoir cité les descriptions d'explorateurs du continent africain (35). Au chapitre 7 , on passe «Du balafon au xylophone », premier pas de «dématérialisation sonore et de raffinement dans la facture », «passage du domaine des bruits à celui des sons purs », qui conduit à «s'abstraire du cercle des données naturelles» (47) en passant par le seuil du résonateur, et celui des intervalles - on s'arrache donc du corps (naturel) pour s'élever vers l'esprit (immatériel, raffiné, abstrait). Au chapitre 8 , «dès lors le banjo nous apparaît comme un stade intermédiaire entre le xylophone et le piano dans l'évolution de l'orchestre afro-américain vers le jazz» (54-55), et permet d'apporter un nouveau type d'indices : les termes banjo, samba, marimba, balafon se retrouvent dans quantité de toponymes sur tout le continent africain (58-60). Avec les vents du chapitre 9, on observe la première appropriation « nègre » d'instruments européens :

« une des qualités essentielles du musicien nègre sera (...) de s'adapter au jeu des instruments européens qu'ils trouvèrent en Amérique, principalement des cuivres, ou plutôt d'adapter ce jeu à leurs profondes (raciales) convenances musicales », afin d' " y vomir toute la violence que mimique et batterie, cependant exacerbées, n'ont pas suffi à épuiser» $(65,64)$.

Le chapitre 10 s'arrête sur les échelles pour montrer "l'existence en quelque sorte inconsciente chez eux d'une gamme type », plus que d'une échelle à proprement parler, et placer «le caractère ambigu de la dominante du mode majeur (...) à l'origine de ces accords de septième diminuée qui se retrouvent toujours dans les chants afro-américains, dans les blues, dans les harmonies du jazz » (70).

Le 11e chapitre est central, puisqu'il ressaisit toute la démonstration généalogique autour de «la voix du nègre » (72). On tient donc le nœud du raisonnement :

«nous pouvons nous demander si la qualité de son organe (vocal), les caractéristiques (naturelles) de son chant n'ont pas eu autant d'influence sur la conformation de sa musique que l'acuité de son sens sur tout ce qui est rythmique et percussion »; en effet, « en lui-même, le nègre est déjà un instrument de musique (...). Il porte en son corps même l'idéal de toute sa musique. Il pouvait donc partir d'Afrique nu : à s'écouter, il écoute les éléments primordiaux de son art. (...) Nous avons devant nous un type de musique dont tout le caractère et toute l'originalité tiennent en quelques procédés techniques, eux-mêmes à un problème de race, et dans ce que pareil problème peut offrir de plus physiologique » (72-73).

Cette voix nègre se déploie, au chapitre 12, dans le choral protestant, opérant «par l'esprit (...) ce mariage de deux races que les usages réprouvent encore dans la chair» (87), ceci par «l'imitation des chorals protestants qu'ils connurent auprès des missions évangéliques - imitation qui alla même jusqu'à l'emprunt direct à la mélodie de ces chorals » (85). On aboutit alors, au chapitre 13, à la «naissance du jazz »:

«Ainsi donc, une fois l'élan donné, par ce premier acte d'annexion qui fit du choral protestant une œuvre afro-américaine, par ce procédé de continuelle syncopation que nous 
montrions inhérent à toute diction nègre et dont le ragtime ne devait être plus tard que la prise de conscience et le grossissement systématique, peu à peu, de transposition en transposition, toute une polyphonie proprement vocale (le spiritual) allait, en se laïcisant, en se matérialisant, passer dans l'usage instrumental. Du reste, le jazz, s'il est une conversation entre instruments, peut s'interpréter également comme une danse de ceux-ci, - et nous savons combien la danse nègre est déjà imprégnée d'habitudes responsoriales, anticipe sur les procédés du concerto (grosso : baroque) » (94).

Parlant de formes élémentaires, on ne manquera d'évoquer l'enquête d'Émile Durkheim sur Les formes élémentaires de la vie religieuse ${ }^{65}$, données à voir chez les peuples primitifs d'Australie. Or, Schaeffner a suivi les cours de Mauss à l'Institut d'ethnologie de janvier 1926 à avril $1927^{66}$, et certaines indications du Manuel d'ethnographie qui concernent les «Arts musicaux: danse, musique et chant, le drame, poésie, prose» (chapitre V: «Esthétique »), présentent des parallèles frappants avec les thèses de Schaeffner, dès cette étude sur le jazz ${ }^{67}$. Pour autant, aucun auteur durkheimien n'est cité dans Le Jazz - ni même aucun ethnologue : seulement des «explorateurs » et des musicographes. Il s'agirait donc seulement d'une affinité, qui expliquerait comment Schaeffner a pu se couler si facilement dans le cadre cognitif et institutionnel du pôle durkheimien. Le texte lui-même suggère d'autres sources : les schèmes qui organisent cette généalogie du jazz - qui, donc, glisse insensiblement vers une généalogie de la musique - ont plus à voir avec le champ musical, ses avant-gardes, ses musicographes et ses rénovations pédagogiques. Le nœud cognitif en est, d'une part, l'opposition «dalcrozienne» entre rythme vital (primitif, africain) et mesure abstraite (civilisée, européenne), et d'autre part, le schème « nietzschéen » du « masque » (trouvé dans la voix chantée, les résonances des matériaux naturels telles que manipulées puis amplifiées par les résonateurs), comme émergeant sans solution de continuité de l'élan corporel et instaurant en même temps une rupture vers le jeu, l'humanité. La méthode est une combinaison de l'intérêt organologique pour les instruments, de l'érudition philologique de l'histoire de la musique et du savoir «muséal» emprunté aux archéologues (Reinach) ou aux folkloristes, mais aussi à l'organologie savante (son étude sur le clavecin est contemporaine de celle sur le jazz) : attention portée à la description matérielle des objets, raisonnement classificatoire, reconstitution des usages et des filiations - notons qu'outre la documentation écrite, Schaeffner a observé les « salles africaines du Musée ethnographique du Trocadéro » (MET) ${ }^{68}$. Quant aux marqueurs de positionnement musical, ils sont désormais modernistes : les références les plus fréquentes sont à Strawinsky et Milhaud.

\section{Investissements académiques}

Le jazz a permis la rencontre de Schaeffner avec Georges Henri Rivière (1897-1985), probablement en $1927^{69}$. Ce dernier est alors intendant des collections artistiques du financier Daniel David-Weill (donateur du Musée d'ethnographie du Trocadéro), et fréquente les salons et loisirs publics de la bourgeoisie parisienne lettrée, et particulièrement les réseaux de collectionneurs, marchands et amateurs d'arts extra-

\footnotetext{
${ }^{65}$ DURKHEIM, 1912.

${ }^{66}$ Cf. JAMIN, in SCHAEFFNER, 1996, 104.

${ }^{67}$ MAUSS, 1947, 86-87.

${ }^{68}$ Cenuroy, SchaEFFner, 1988 (1926), 39.

${ }^{69}$ Cf. SCHAEFFNER, 1980 (1964).
} 
européens ${ }^{70}$. Dénué de diplôme universitaire tout comme Schaeffner, il suit les cours de l'École du Louvre de 1925 à 1928. C'est alors que Christian Zervos, responsable des Cahiers d'art auxquels Rivière a collaboré dès 1924, commande à ce dernier un article sur l'art pré-colombien, et l'envoie pour ce faire au MET. Zervos a en effet été séduit par la visite de l'exposition «Yves Tanguy et objets d'Amérique» organisée par la galerie surréaliste au début de 1927 : quand le succès de l'«art nègre » s'épuise, ce nouveau créneau apparaît comme un renouvellement possible. Rivière se montre enthousiaste à son tour, et décide avec Georges Salles, alors conservateur au Musée du Louvre, de monter une grande exposition. Ils obtiennent l'accueil du Pavillon de Marsan (Musée des arts décoratifs), qu'ils remplissent de plus de 1200 objets venus du MET et de 18 collections privées - dont celles de Marcel Mauss, du vicomte de Noailles et des surréalistes Roland Tual et André Breton. La famille Salatini met son hôtel à la disposition de la petite équipe que Rivière rassemble pour classer les objets et réaliser les cartels et le catalogue : Schaeffner, Georges Bataille (1897-1962) et Alfred Métraux (1902-1963), le seul du groupe à pratiquer déjà l'ethnographie et à être en contact avec les ethnologues professionnels de l'Institut d'ethnologie de Paris. La classification est organisée par pays, puis par matériau, et la présentation emprunte au modèle esthète qui a prévalu pour 1 ' art nègre ${ }^{71}$. L'exposition est un succès public, et se trouve appréciée notamment par Paul Rivet, qui vient d'être nommé à la chaire d'anthropologie du Museum national d'histoire naturelle, et par contrecoup, directeur du MET dont il a obtenu le rattachement à sa chaire en vue d'une réorganisation des salles et des collections. Pour réaliser cette dernière, il nomme Rivière sous-directeur du MET car il en apprécie les compétences médiatiques et organisationnelles et le capital social. Peu après, celui-ci sollicite Schaeffner afin de créer un département d'organologie, c'est-à-dire une simple salle d'exposition spécifique, grâce à la récupération des collections d'instruments de quelques personnes privées et surtout du Conservatoire ${ }^{72}$.

Schaeffner quitte alors les rédactions du Ménestrel et de La Revue Musicale, mais conserve, parmi ses activités rémunérées dans le champ musical, le poste de secrétaire artistique de l'Orchestre de Paris obtenu dès sa création en octobre $1928^{73}$. Autrement dit, il ne s'agit pas d'un désinvestissement du champ musical, mais d'une extension de son domaine à l'ethnographie : présenter « la musique », en des états certes situés aux deux extrémités de «l'évolution». De fait, les années qui suivent sont marquées pour Schaeffner par des activités et publications dans les deux domaines.

Du côté musicologique, la Société française de musicologie, créée en 1917 et dotée de sa Revue de musicologie en 1922, est reconnue d'utilité publique en 1931. Si seules les universités de Strasbourg et de la Sorbonne proposent des diplômes spécifiques d'histoire de la musique dans l'entre-deux-guerres, les institutions délivrant des thèses à des

\footnotetext{
${ }^{70}$ Sur Rivière, $c f$. CHIVA, 1985 ; CHEYRONNAUd, 1987 ; LEROUX-DHUYS, in RIVIÈRE, 1989 ; LAURIÈRE, 2003.

${ }^{71}$ Les récits ultérieurs affirmeront que cet esthétisme, opposé aux pratiques développées ultérieurement, visait une sorte de coup publicitaire destiné à valoriser les collections du MET, délaissées depuis le début du siècle, auprès des ethnographes (LAURIÈRE, 2003).

${ }^{72}$ En 1925, le poste de conservateur du Musée instrumental du Conservatoire était supprimé, et les collections placées sous la responsabilité du conservateur de la bibliothèque du Conservatoire, ie délaissées (GÉTREAU, 1993). Il n'est donc pas impossible que la création du département soit en partie sous-tendue par une démarche de sauvegarde: en «1927 ou 1928 (...). Il y avait Chantavoine (directeur du Conservatoire) qui voulait se débarrasser de tous les instruments exotiques (...). Alors Rivière m'a dit "Oh! Vite, précipite-toi". Alors j'ai tout pris. Ça a été le début du département» (DUVELLE, 1982b, 373).

${ }^{73}$ Il tient toutefois la rubrique «Lettera da Parigi », semble-t-il irrégulière, dans La Rassegna Musicale de février 1928 à juillet 1934, et publie ponctuellement dans d'autres revues. À l'Orchestre de Paris, Schaeffner est chargé de la rédaction des notices des programmes et des négociations avec les éditeurs afin d'obtenir les partitions au moindre coût. Cf. F.S., Dossiers de travail, 9, « OSP ».
} 
musicologues se font plus nombreuses, contribuant à former une jeune génération « savante », sans compter les diplômes non universitaires de la Schola Cantorum ${ }^{74}$. Dans ce contexte, la réédition en 1931 du Dictionnaire de musique d'Hugo Riemann, refondu sous la direction de Schaeffner, tente de promouvoir le registre savant dans le domaine musicographique, sans effets immédiats semble-t-il, si ce n'est celui de doter Schaeffner d'une autorité savante auprès de ses pairs. Dans le court avant-propos, il résume ainsi les points saillants de la nouvelle doctrine, à la fois «puriste » et «néoclassique » : écartement de nombreux professeurs, interprètes et amateurs, considérés désormais comme secondaires face aux compositeurs professionnels; «rééquilibrage » des périodes au détriment du $\mathrm{XIX}^{\mathrm{e}}$ siècle, allemand, et au profit des périodes anciennes et classiques, françaises, anglaises et italiennes, ainsi que de la période contemporaine ${ }^{75}$. Par ailleurs, Schaeffner collabore, pour cet ouvrage, avec les jeunes musicographes de la SFM, par contacts individuels plus que par investissement de l'institution toutefois ${ }^{76}$ : André Tessier en est le secrétaire général au moment de sa mort, en juillet 1931; Marc Pincherle devient secrétaire général à la suite de Tessier, jusqu'en mars 1935; Yvonne Rokseth est membre du conseil d'administration à partir de janvier 1933.

La même année 1931, Schaeffner publie sa monographie «savante » sur Strawinsky, la deuxième après celle de Schloezer en 1929 ( $c f$. supra). Il en a conservé douze comptes rendus élogieux, parus entre le 24 juin 1931 et le 2 janvier 1932, sous les plumes de critiques ou de compositeurs en vue. Significativement, on retrouve les mêmes qualifications de l'un à l'autre, qui valorisent ce caractère «savant » : rigueur et technicité de l'étude ; appui sur les entretiens avec le compositeur. Dans le même sens, Pierre-Octave Ferroud regrette une prose «diffuse, laborieuse, empreinte même de gaucherie»; et Paul Landormy l'oppose à l'inverse aux divagations philosophiques «aventureuses » de l'ouvrage de Schloezer. De même, Gabriel Marcel s'excuse, dans une lettre du 7 juillet 1931, de ne pouvoir rendre compte de ce livre « si solide et si plein » pour la NRF faute de temps, tout en soulignant qu'il aurait été négatif car "nulle part je n'y vois l'ombre d'un jugement», ni l'établissement d'une «hiérarchie entre ses auvres (de Strawinsky). Ceci me paraît inadmissible ${ }^{77}$.

Du côté ethnologique, Schaeffner et surtout Rivière élaborent au sein du MET une muséologie contextualiste, ie ni esthète ni évolutionniste, qui présente les objets à l'aide de scènes reconstituées, de vitrines comparatives et de cartels expliquant leurs usages habituels, sur la base de classifications fondées sur leurs origines géographiques (saisies au sein d' " aires culturelles ») ${ }^{78}$. Quand le second visite les musées d'ethnographie étrangers, notamment en Europe du nord, le premier publie les principes de cette rénovation, au sujet des instruments, dans trois articles pour la revue Documents que mène Georges Bataille ${ }^{79}$, pour le premier numéro du Bulletin du MET et pour La Revue Musicale ${ }^{80}$. En 1931, l'équipe du MET contribue à l'Exposition coloniale de Vincennes (Schaeffner y présente

\footnotetext{
${ }^{74}$ Cf. VANHULST, 2000.

${ }^{75}$ RIEMANN, 1931, VII-VIII.

${ }^{76}$ Il ne sera assidu aux séances de la SFM qu'à partir de 1942 ( $c f$. les comptes rendus publiés par la Revue de Musicologie).

${ }^{77}$ F.S., Dossiers de travail, 6.

78 JAMIN, 1982 ; 1986 ; RiviÈRE, 1989 ; DiAS, 1991 ; CHEYRONNAUd, 1997 ; SHERMAN, 2004. « Nous montrerons ces objets, dans nos futures salles du Trocadéro, avec le maximun de documentation. (...) Cet instrument de musique, une photographie vous le montrera en usage, une portée musicale vous en donnera l'étendue, un disque voisin vous en fera entendre le son »(Georges Henri Rivière, "À propos de l'art nègre », Le Figaro Esthétique, août 1931, cités in LAURIÈRE, 2003, 64).

${ }^{79}$ Sur celle-ci, $c f$. JAMIN, 1999.

${ }^{80}$ SCHAEFFNER, 1929, $1931 \mathrm{~b}$ et 1932.
} 
par exemple des danses de masques dogons) ${ }^{81}$, et participe à la mission de collecte «fondatrice» Dakar-Djibouti (Schaeffner s'y rend de Bandiagara le 18 août 1931 à Yaoundé le 19 décembre 1932). Au retour de Schaeffner, le « département » qu'il dirige est rebaptisé «d'ethnologie musicale» et doté d'un phonographe et de collections d'enregistrements (dont ceux réalisés durant l'Exposition coloniale), qu'il fait écouter au cours de séances d'audition commentées les samedis après-midi. En 1933 et 1934, il accueille Curt Sachs, organologue allemand réputé, en exil, qui travaille sur les instruments en provenance de Madagascar ${ }^{82}$, et Claudie-Marcel Dubois, à qui Sachs conseille l'étude des instruments en provenance d'Inde ancienne, avant qu'elle se spécialise dans le folklore français - elle intègrera à ce titre le département de folklore du Musée des arts et traditions populaires (MATP) à sa création en 1937, et y établira en 1939 le Service d'ethnographie musicale ${ }^{83}$. Les collections s'enrichissent au gré des dons (notamment du Conservatoire et du Musée des antiquités nationales) et des missions (en 1935, Schaeffner et Griaule collectent du Sahara au Soudan). Dans le même temps, la plupart des collaborateurs du MET participent aux enquêtes collectives (à partir de 1935) et à la rédaction des articles de l'Encyclopédie française que dirige Lucien Febvre depuis 1932 (et qui mobilise l'ensemble du pôle académique durkheimien ${ }^{84}$ ). En 1937, la réorganisation du Palais du Trocadéro et de ses musées aboutit, à l'occasion de l'Exposition universelle, à la création du Musée de l'homme (que rejoint Schaeffner) et du MATP, séparant durablement les domaines lointains et proches. Certains des collaborateurs sont alors intégrés statutairement au récent CNRS, comme Leiris ou Schaeffner en 1941, ce dernier recrutant Gilbert Rouget comme assistant. Au total, le dispositif de savoir qui se stabilise entre musées et missions est donc centré sur la collecte et la classification des objets indigènes - et c'est de ces opérations cognitives routinières, matérialisées en agencements d'objets et en accumulations de fiches, que se démarqueront, surtout après la guerre, les observations de rituels et les recueils de schèmes classificatoires et de mythes, considérés comme donnant accès au fonds mental partagé de chaque société étudiée, voire de « la civilisation africaine ».

Or, l'ethnologie et le folklore musicaux connaîtront moins ce déplacement d'intérêt de la culture matérielle vers la culture symbolique, qui sera d'ailleurs accompagné par le déclassement des espaces muséaux au profit du «terrain». D'un côté, ils resteront largement centrés sur l'aspect «technique» de la collecte et de la description des instruments. De l'autre côté, le graphocentrisme musicologique reviendra en force, au point d'apparenter les travaux à une simple musicologie qui s'annexerait des pratiques insolites - via l'opération cruciale (et possiblement utile, précisons-le), et pourtant alors inaperçue comme telle le plus souvent, de la transcription graphique, «sur portées » puis sur spectrogrammes, de performances vives ou enregistrées et de savoir-faire musiciens qui s'organisent sans techniques de notation, et tout ce qu'elles impliquent ${ }^{85}$. On peut donc se demander si l'organologie, telle que cadrée par la somme programmatique de Schaeffner,

${ }^{81}$ DE L'Estolle, 2001. $C f$. le texte de sa présentation et le commentaire qu'en fait Jean Jamin : SCHAEFFNER, 1996 (1931).

${ }^{82}$ SACHS, 1938 (dans la collection dirigée par Marcel Mauss). Peu après son arrivée en France, il avait rédigé et publié un précis de muséologie musicale (SACHS, 1934). Sachs est, avec Erich von Hornbostel, le fondateur de l'organologie «scientifique» allemande, inspirés par le belge Victor Mahillon. Schaeffner leur emprunte directement méthodes et hypothèses, tout en prétendant démarquer son propre système de classification, fondé sur les propriétés matérielles des instruments et non leurs principes de résonance (SCHAEFFNER, 1931b).

${ }^{83}$ Marcel-Dubois, 1941. À son sujet, Cheyronnaud, 2003 ; Cheyronnaud, Cuisenier, 1989.

${ }^{84}$ FABRE, 1997 ; WEBER F., 2002 ; MULLER, 2003. Schaeffner ne fait pas partie des équipes d'enquêteurs, qui fourniront surtout le futur musée des ATP.

${ }^{85}$ Cf. Cheyronnaud, 1997. 
constitue une réelle sortie de ce paradigme «graphocentriste », malgré la rupture qu'elle réalise avec le centrement intellectuel sur la partition.

\section{Le détour ethnologique}

En effet, à bien des égards, l'Origine... est une extension, une systématisation et une révision du Jazz paru dix ans plus tôt. Quelques points d'analyse sont revus, les comparaisons sont largement développées et affinées, mais on y retrouve les mêmes schèmes organisateurs. Pour mesurer la réalité et la relativité conjointes de cet approfondissement, on peut s'arrêter d'abord sur la contribution de Schaeffner à L'Encyclopédie française consacrée au jazz ${ }^{86}$. On y observe combien elles sont essentiellement le produit des opérations de classement et de comparaison que permet le dispositif muséal. C'est lui qui conduit à revenir en partie sur le racialisme qui organisait $L e$ $J a z z$ : il évoque l'importance des musiques amérindiennes, oubliées dans les généalogies du jazz existantes, et un «métissage auquel il est peu probable qu'aucune musique d'Amérique n'ait échappé »; et conclut que «vis-à-vis de la musique moderne d'Europe le jazz a joué le rôle d'un folklore. Or le jazz se présente en réalité comme une musique "fabriquée" et à ce titre est cosmopolite ${ }^{87}$. Cependant, cette révision est fondée sur la dissociation entre code musical (règles «mentales» de combinaison des sons, transposables graphiquement) et performance (pratique «corporelle» in situ), clivage «scolastique» qui va structurer la discipline. Ainsi la performance est produite par la rencontre entre le code musical et les «habitudes corporelles» des interprètes : celles-ci sont irréductibles au code, mais réductibles aux «données raciales ». Dès lors, il n'y a pas de contradiction logique apparente à affirmer que le jazz est à la fois métissé (du point de vue des codes musicaux) et «nègre» (du point de vue des «habitudes corporelles» d'interprétation, qui fondent l'essence du jazz) :

«Le jazz est un "ersatz" de folklore ; toutefois, par la profondeur de son influence, par

l'accent en quelque sorte de vérité qui s'en dégage, par la sincérité aussi de ceux qui le firent

(et nous pensons d'abord aux nègres), le jazz est la musique d'un peuple ${ }^{88}$.

L'Origine... vient déployer cette clarification qui a toutes les apparences d'une formation de compromis sur fond de clivage cognitif, car elle permet de tenir ensemble l'universalité métaphysique de l'entité «musique» et la diversité empirique de ses actualisations. L'ethnographie, c'est-à-dire moins l'emploi de méthodes et de systèmes de questionnements spécifiques que l'étude des peuples exotiques, sert ainsi à ramener le « primitif », le corps et l'oralité, au cœur du phénomène musical. L'opération est, si l'on peut dire, temporalisée plus qu'historicisée : le primitif est l'élémentaire, le civilisé est le complexe ; et ces deux pôles sont reliés à la fois par la continuité d'une évolution, synonyme de complexification, et celle d'une essence, identifiée au plus « simple » ${ }^{89}$. Avec le pôle «élémentaire », la musique apparaît en quelque sorte dans la nudité de son essence, avant que des habits de plus en plus élaborés viennent la revêtir, voire la dissimuler : le corps et l'oral ont été refoulés par la musique «pure », jusqu'à se perdre dans l'abstraction des analyses de partitions.

${ }^{86}$ Son autre contribution est un condensé de l'Origine... : SCHAEFFNER, 1935b. Il a aussi publié un premier exposé du point central de ses perspectives dans une revue musicale, Le Ménestrel (SCHAEFFNER, 1934).

${ }^{87}$ SCHAEFFNER, 1935a.

${ }^{88}$ Ibid.

${ }^{89}$ SCHAEFFNER, 1936, 8. Nous indiquons désormais les numéros des pages d'où sont extraites les citations à leur suite, entre parenthèses. 
En termes de positionnement ethnologique, Schaeffner se voit dès lors contraint de se démarquer de la «théorie des cycles d'évolution» que Georges Montandon, qui devient alors une figure du pôle anti-durkheimien, a présentée pour la première fois en 1919, précisément dans un catalogue des collections d'instruments de musique du Musée d'ethnographie de Genève. Montandon y contestait les classifications de Mahillon, Hornbostel et Sachs sur lesquelles s'appuiera Schaeffner, et axait «l'établissement des chaînes généalogiques» autour du postulat que la «grande coupure dans le développement » de la musique serait intervenue au $\mathrm{XVI}^{\mathrm{e}}$ siècle avec «l'emploi dès lors, des lois de l'harmonie (le système tonal). (...) C'est que, à notre jugement, la musique est par excellence l'art $d u$ christianisme ${ }^{90}$. Pour appuyer sa démarcation, Schaeffner cite longuement, en note, Marcel Mauss (8). Ces références sont les seules faites à des ethnologues qui dépassent la simple mention de sources. C'est qu'elles ne structurent pas l'argumentation, mais sont rendues nécessaires par l'anticipation de malentendus probables, au vu du caractère généalogique de l'étude et de sa conséquence : l'absence des méthodes et problématiques que ses collègues et alliés construisent au même moment. Plus, la reconstitution de filiations d'objets classés en fonction de leur morphologie est précisément le type d'option contre lequel s'est établie la rupture muséologique initiée par Rivière : les objets muséaux sont désormais considérés comme des «types culturels » ou des « objets témoins » d'une culture ou une aire culturelle, en opposition aux pratiques antérieures qui en faisaient les témoignages d'un stade d'évolution ${ }^{91}$. On comprend ainsi que la (courte) pique contre Montandon, théoricien des «ethnies » (terme repris à Vacher de Lapouge) et de leurs degrés de civilisation dont le maître-livre vient de marquer la rupture avec ses premiers soutiens durkheimiens ${ }^{92}$, soit une précaution nécessaire : le projet généalogique et les schèmes anti-rationalistes qui organisent les analyses de l'Origine... pourraient entretenir l'ambiguïté.

De fait, le postulat qui ouvre l'étude est repris à la généalogie nietzschéenne : à l'origine de la musique instrumentale n'est pas la raison imitative (la voix se faisant instrument pour imiter la nature, puis imitée par les instruments de musique fabriqués), mais le corps dansant qui se fait «masque», «débordement» de la nature - et ainsi, la musique instrumentale est dès «l'origine » indépendante de la musique vocale (non traitée dans l'ouvrage). Le schème est décliné à de multiples reprises, et développé par l'idée d'une «extériorisation» de la musique (du corps vers les instruments, mais aussi, plus loin, du rythme attaché au corps vers la mélodie vocale puis l'harmonie « raisonnante») - par exemple, on voit l'instrument déborder le corps, puis être débordé lui-même avec la résonnance, le maintien du son, alors la musique «s'extériorise » (53). L'opérateur nodal de cette généalogie est donc le résonateur. Par lui, les possibilités naturelles de la voix et des matériaux sont «débordés », ce qui les rapproche du masque tragique, qui marque la naissance du drame selon Nietszche, et fait de la musique un phénomène essentiellement «baroque », donc non classique, tempéré, rationalisé... (25-26). Cependant, le masque apparaît bien plus «tôt », dès qu'une intention spécifique vient organiser la production de sons : la musique émerge de la danse rituelle, quand les sons que produisent les gestes rythmés du danseur sont considérés pour eux-mêmes, amplifiés par de nouveaux gestes puis des ustensiles créés à cet effet, puis développés au delà de la continuité avec le geste dansant. En effet, les rituels primitifs confondent religion, danse et musique, si bien qu'il est impossible de les dissocier, et encore moins de les hiérarchiser (ce que le «drame total»

\footnotetext{
${ }^{90}$ Montandon, 1919, 6.

${ }^{91}$ DIAS, 1991. Sur les attendus implicites de ce nouveau rapport aux objets muséaux, $c f$. BENSA, 2003.

${ }^{92}$ L'Ologenèse culturelle. Traité d'ethnologie cyclo-culturelle et d'ergologie systématique (publié lui aussi chez Payot, en 1934) est la reprise développée des thèses proposées dans le catalogue de 1919 (FABRE, 1997).
} 
wagnerien semblait viser, $c f$. infra), et qu'aux origines de la musique instrumentale se trouve «le pied du danseur et non la main du musicien » (34). Le «premier» instrument est donc le corps du danseur, «suivi » de près par les sonailles (objets attachés aux bras ou aux jambes et qui tintent avec les gestes du danseur), qui font passer la gestuelle du côté de l'intention musicale (et non de l'intention plastique) - titre du deuxième chapitre : «Des sonailles aux castagnettes ». Les différenciations, ici comme dans toute la démonstration, sont fondées sur des modes de vibration (l'association entre des matériaux et des gestes), et ce principe classificatoire, d'ordre «logique», détermine les filiations reconstituées indépendamment des histoires concrètes des instruments (inaccessibles à l'observation dans la plupart des cas). Ceci produit, pour le non spécialiste tout du moins, une impression d'arbitraire typique de la situation de skolè. Elle est liée à l'absence de preuves positives : Schaeffner passe directement de la comparaison d'objets (ou de leurs fiches descriptives) rassemblés, à plat et simultanément, dans l'espace du musée, à l'indexation de chacun d'entre eux sur un fil d'évolution constitué par un raisonnement logique, et non par des méthodes de datation ni même des récits décrivant les origines de chaque instrument.

Quelques propositions nodales organisent les autres, qui convergent vers la critique du graphocentrisme de la musicologie. Premièrement, il y a «deux sources » à la musique, définies transversalement à toute classification des races ou des cultures, et qui diffèrent en «essences» et non en «valeurs»: non pas la polyphonie (européenne) vs le primitif ou l'oriental, mais «la double existence, à l'origine, d'une musique née du chant et d'une autre née du corps, ou si l'on veut: avec la danse»(309-310). Deuxièmement, la musicologie gagne à ajouter les instruments à ses documents habituels (partitions et textes) ${ }^{93}$, mais aussi à faire converger ces traces du passé vers la recherche de ce qui laisse le moins de trace, et qui pourtant se trouve au plus près de ce qu'est la musique : non pas des notes sur papier, mais des performances vives. Il s'agit des «conventions tacites» (autrement dit, les «habitudes corporelles» déjà rencontrées) qui actualisent les possibilités des instruments, les directives de la partition et les principes des théories esthétiques (303).

Troisièmement, le graphocentrisme ${ }^{94}$ limite considérablement l'étude de la musique, en excluant «ce qui dans la musique est expression et action » :

«Combien il est présomptueux en effet d'envisager la musique sous le seul aspect d'une

"littérature", et nous voulons dire : d'une littérature écrite, alors que tant d'exemples, même contemporains, prouvent combien cette écriture est imparfaite, combien cette orthographe est inapte à reproduire la complexité et le mouvement d'un langage qui est moins lu que "parlé". Et là n'apparaît que trop l'inexpérience d'une musicologie classique manquant de sympathie envers toute musique qui se fait sous ses oreilles - que ce soit musique moderne ou musique se parlant encore sous d'autres latitudes. À se pencher trop sur des musiques mortes ou ne se jouant plus naturellement, ne perd-on point le sens de ce qui dans la musique est expression et action ? »(369-370, il souligne).

Cette théorie alternative de la musique ne débouche pourtant pas sur l'étude empirique de ces pratiques « expressives » et « actives » qu'elle paraît appeler. À cet égard, l'annonce de futures publications «de caractère totalement différent» (12), basées sur des matériaux recueillis lors des deux missions chez les Dogons, montre qu'il envisageait le traitement des

${ }^{93}$ Car « de tous les vestiges pouvant nous renseigner sur un art qui tend à s'évanouir, et cela dès l'instant même où il jaillit faute d'écriture capable d'en sauvegarder la totalité, l'instrument est encore ce dont nous avons gardé le plus de témoignages certains à travers le temps » (370).

${ }^{94}$ Il n'emploie pas ce terme, mais nous l'utilisons pour faire court, avec cette précision toutefois : la centralité accordée à la partition entraîne selon lui une fuite dans l'abstraction, un éloignement de la pratique musicale concrète ; le graphocentrisme constitue pour nous une grille de perception tout aussi « concrète » et «sensible » que les autres modes d'appréhension des faits musicaux. 
performances rituelles à partir d'une reconstitution de codes musicaux, chorégraphiques et symboliques que le rite ferait converger par un jeu de «correspondances formelles» arbitraires ${ }^{95}$. Ainsi, le plan annoncé comporte: «un tableau exhaustif des types d'instruments » employés dans la région de Sanga et de «tous les usages auxquels ces instruments sont liés»; une «musicologie comparée» destinée à «disposer ces instruments selon leurs âges relatifs et aboutir à une chronologie vraisemblable »; un « répertoire de leurs batteries»; enfin, la description des musiques et danses effectuées lors de rituels funéraires, centrée sur la question des «correspondances formelles » entre musiques et danses, et entre performances rituelles et figurations (de mythes, de personnages, d'objets) qu'elles réalisent. Ces quelques dernières pages de l'introduction montrent un Schaeffner in fine démuni pour traiter de la logique pratique des performances : la «rencontre » entre les différents codes reconstitués, si elle est analysée comme contingente, lui paraît simplement «arbitraire», sans logique ni consistance propres, et semble renvoyer en réalité aux questions esthétiques sur les rapports entre les différents arts de la scène, qu'avaient renouvelées le «drame total » wagnerien puis, autour du ballet, l'avant-garde musicale des « Six ».

\section{Conclusion}

C'est vers l'âge de 34 ans que Schaeffner investit la discipline ethnologique en voie d'académisation, en son pôle durkheimien. Auparavant, il s'est constitué une position de musicographe, initiée via les réseaux scholistes puis établie au pôle moderniste et savant qu'incarnent respectivement La Revue Musicale et la Société française de musicologie. Ces déplacements aboutissent à l'accès puis au maintien d'une double position institutionnelle dont les caractéristiques renvoient aux configurations de chaque discipline : quand il préside une SFM (1948-1968) relativement excentrée tant dans le monde académique que dans le champ musical, il dirige un département du musée de l'Homme qui mêle recherche et muséologie, et qui occupe une place à la fois privilégiée au sein du secteur muséal, et de plus en plus marginale au sein de la discipline dans son ensemble. Son statut de fondateur du département d'ethnologie musicale et le désintérêt des musicologues pour les musiques non savantes et européennes expliquent qu'il soit cité aujourd'hui comme ethnologue bien plus souvent que comme musicologue - et inversement, échouant à s'imposer aussi bien que ses collègues du musée de l'Homme dans la discipline ethnologique, il insistera vers la fin de sa vie sur ses écrits musicologiques ${ }^{96}$. Cependant, cette double position ne produit pas d'écart ou de tension intellectuelle entre les deux investissements : l'ethnologie fournit des matériaux et des décalages critiques à des propositions qui s'inscrivent, intellectuellement, dans le champ musical.

Qui plus est, les schèmes intellectuels qui organisent les analyses tant musicales qu'ethnographiques renvoient à des socialisations antérieures à l'activité ethnologique, en prise directe avec les débats et les transformations de ce même champ musical au sortir de

95 Jean Jamin a publié et présenté une introduction à cet ouvrage jamais abouti dans le $\mathrm{n}^{\circ} 177-178$ de L'Homme, avec une postface de Bernard Lortat-Jacob (SCHAEFFNER, 2006). Ce dernier commente un manuscrit correspondant à cette introduction, limité de façon significative à des considérations strictement organologiques (ce qu'indique le titre, différent de celui annoncé en introduction : Les instruments de musique des Dogons). Ses analyses rejoignent les nôtres.

${ }^{96}$ En 1980 paraît un recueil d'articles intitulé Essais de musicologie et autres fantaisies (SCHAEFFNER, 1980). Outre les rééditions (Lettres à Peter Gast, Origine..., Le Jazz, Les Kissi - ce court compte rendu des missions des années 1940 était paru en 1951, il est repris dans SCHAEFFNER, 1990), les éditions posthumes comptent une reprise augmentée des Essais (SCHAEFFNER, 1998a) et la correspondance avec Boulez (BOULEZ, SCHAEFFNER, 1998). 
la Première Guerre mondiale. Ce qu'on peut appeler le purisme sensualiste de Schaeffner s'est forgé auprès du cercle Baruzi, des musicographes scholistes et des thématisations de la gymnastique rythmique et de la musique ancienne. La critique du graphocentrisme de la musicologie s'ancre ainsi dans le champ musical lui-même, plus précisément dans le primitivisme et l'anti-rationalisme que figure alors un Strawinsky - et Schaeffner lui-même affirmera que c'est moins le jazz que la musique moderne qui l'a conduit à s'intéresser aux musiques «élémentaires ${ }^{97}$. L'ethnologie viendra la déployer et la fonder par une généalogie «nietzschéenne» de la musique. Mais en quittant le graphocentrisme, Schaeffner rencontre le muséocentrisme de la discipline ethnologique telle qu'elle s'institue dans les années 1930, spécialement en son secteur musical : les mêmes schèmes servent alors à orienter la manipulation des objets (collecte, classification) héritée des musées d'archéologie, d'art et de folklore vers une restitution de leurs usages « vivants ». C'est ainsi qu'on peut rendre compte du caractère en partie insituable de l'Origine...: les minutieuses descriptions organologiques y sont entièrement décontextualisées, pour servir à la fois un projet universaliste (la musique est une, quelles que soient ses formes et les sociétés qui la pratiquent) et une philosophie esthétique qui s'attache à déterminer, par une fiction généalogique, l'essence de la musique. La critique du graphocentrisme et le passage par l'organologie, qui paraissent miner les principes du champ musical et contrer l'ethnocentrisme des études de folklore, permet au contraire de sauver l'essentiel, c'est-àdire la musique comme essence intemporelle : que la musique se situe dans la partition ou dans la performance, elle est une et existe indépendamment de ses actualisations - élémentaires, pures ou raffinées, savantes ou populaires - comme entité séparée ; et porter le regard sur la diversité de ses réalisations dans le temps et dans l'espace ne peut que la magnifier un peu plus encore. Après tout, ne trouve-t-on pas «partout» des objets susceptibles d'être qualifiés d'instruments de musique? Musicologie et ethnologie musicale peuvent ainsi être pratiquées sans solution de continuité, avec le sentiment de servir un même projet - un même fétiche : La Musique.

Olivier RouEFF o.roueff@free.fr

\section{Bibliographie}

BARNeS A., 1924, L'art nègre et l'Amérique, Les Arts à Paris, 9, 2-5.

BARUZi JEAN, 1924, Saint-Jean-de-la-Croix et le problème de l'expérience mystique, Paris, Félix Alcan.

BARUZi Joseph, 1904, Le Rêve d'un siècle, Richard Wagner et Victor Hugo, Paris, Calmann-Lévy. BARUZI JosePH, 1911, La Volonté de métamorphose, Paris, Grasset.

BAUER M., 1924, L'influence du «jazz-band », La Revue Musicale, 1, 31-36.

BELVIANES M., 1982, L'homme qui nous faisait aimer ce qu'il aimait, Revue de Musicologie, Numéro spécial « Les fantaisies du voyageur. XXIII variations Schaeffner », 68, 1-2, 381-386.

97 «(Debussy et Ravel) m'avaient ouvert les oreilles et rendu sensible à un ordre de sonorités qui ne se percevait guère dans la musique classique. Ensuite était venu Strawinsky, avec le Sacre du Printemps, première œuvre que j'aie entendue de lui, en 1914 » (SCHAEFFNER, 1980 (1964), 135). Il faut relever qu'à l'inverse d'un Schloezer, qui portera les élaborations théoriques de cet état du champ musical spécifique à l'entre-deux-guerres jusqu'aux frontières du «structuralisme sériel » de la période suivante ( $c f$. DONIN et KECK dans ce même numéro), il restera durablement ancré dans ses premières conceptions, et ce malgré son soutien et son dialogue avec l'avantgarde boulezienne - Strawinsky et Debussy, comme inventeurs du modernisme en musique approchés par l'analyse des sources et des subjectivités créatrices, demeureront les thèmes quasi-exclusifs de ses travaux et prises de position dans le champ musical. 
Bensa A., 2003, Les musées, les individus et l'histoire, Arquivos do Centro cultural Calouste Goulbenkian, XLV, 5-22.

BENSA A., FASSIN E., 2002, Les sciences sociales face à l'événement, Terrain, 38, 5-20.

BerChtold A., 2000, Émile Jaques-Dalcroze et son temps, Lausanne, L'Âge d'Homme.

Blumenson M., 1979, Le Réseau du Musée de l'Homme: les débuts de la Résistance en France, Paris, Seuil.

Boulez P., SchaeffNer, A., 1998, Correspondance 1954-1970 (présentée et annotée par PereIRA DE TugnY R.), Paris, Fayard.

Bourdieu P., 1998, Méditations pascaliennes, Paris, Seuil.

Cendrars B., 1921, Anthologie nègre, Paris, Éditions de la Sirène.

Charle C., 1998, Paris fin de siècle. Culture et politique, Paris, Seuil.

Cheyronnaud J., 1986, Mémoires en recueils. Jalons pour une histoire des collectes musicales en France, Montpellier, Office Départemental d'Action Culturel.

Cheyronnaud J., 1987, À la scène comme au sanctuaire. Le music-hall et la grand-messe, Ethnologie Française, 1, 46-52.

CheYronNAud J., 1991, «Éminemment français ». Nationalisme et musique, Terrain, 17, 91-104.

Cheyronnaud J., 1994, La musique en ses idiomes. Projet d'ethnographie musicale en 1853, Gradhiva, 16, 93-108.

Cheyronnaud J., 1995, Poétique sonore de la République, Ethnologie Française, 25, 4, 581-589.

Cheyronnaud J., 1997, Ethnologie et musique : l'objet en question, Ethnologie Française, 27, 3, 382-393.

Cheyronnaud J., 2003, Musique, politique, religion. De quelques menus objets de culture (préface de FABIANi J.L.), Paris, l'Harmattan.

Cheyronnaud J., Cuisenier J., 1989, Claudie Marcel-Dubois (1913-1989), Ethnologie Française, $19,4,325-328$.

ChIMÈNes M., 2004, Mécènes et musiciens : du salon au concert à Paris sous la IIIe République, Paris, Fayard.

Chiva I., 1985, George Henri Rivière : un demi-siècle d'ethnologie de la France, Terrain, 5 (article téléchargeable sur le site Internet : http://terrain.revues.org/document2887.html/).

Cocteau J., 1918, Le Coq et l'arlequin. Notes sur la musique, Paris, La Sirène.

Ceeuroy A., SchaefFner A., 1926, Romantisme du jazz, La Revue Musicale, 11, 221-224.

Ceeuroy A., SchaeffNer A., 1988 (1926), Le Jazz, Paris, Jean-Michel Place.

D’Harcourt R., D'Harcourt M., 1925, La musique des Incas et ses survivances, Paris, Librairie orientale P. Geuthner.

D'Indy V., 1903, Cours de composition musicale, Paris, A. Durand.

DAUBRESSE M., 1910, La gymnastique rythmique, SIM, 587-603.

De L'Estoile B., 2001, « Des races non pas inférieures, mais différentes » : de l'exposition coloniale au Musée de l'Homme, in BlancKaert C., (ed.), Les politiques de l'anthropologie. Discours et pratiques en France (1860-1940), Paris, l'Harmattan, 391-473.

DiAs N., 1991, Le Musée d'ethnographie du Trocadéro, 1878-1908 : anthropologie et muséologie en France, Paris, CNRS.

Dolleans E., 1954, Jean et Joseph Baruzi, L'Actualité de l'Histoire, 8, 31-34.

DuCHESNEAU M., 1996, La musique française pendant la guerre 1914-1918. Autour de la tentative de fusion de la Société Nationale de Musique et de la Société Musicale Indépendante, Revue de Musicologie, 82, 1, 123-153.

Duchesneau M., 1997, L'avant-garde musicale et ses sociétés à Paris de 1871 à 1939, Liège, Mardaga.

DURKHEIM É., 1912, Les Formes élémentaires de la vie religieuse : le système totémique en Australie, Paris, Félix Alcan. 
Duvelle C., 1982, Rencontre avec André Schaeffner (1980), Revue de Musicologie, Numéro spécial «Les fantaisies du voyageur. XXIII variations Schaeffner », 68, 1-2, 366-380.

FABIANI J.L., 1997, Controverses scientifiques, controverses philosophiques. Figures, positions, trajets, Enquête, 5, 11-34.

FABIANI J.L., 2002, Le public et sa légende, in ETHIS E., Avignon, le public réinventé. Le Festival sous le regard des sciences sociales, Paris, La Documentation Française, 31-51.

FABRE D., 1997, L'ethnologie française à la croisée des engagements, in BOURSIER J.Y., (ed.), Résistants et résistance, Paris, l'Harmattan, 319-400.

FASSIN E., 1998, Play it again, Sartre ? New Dreyfusards in Search of a New Dreyfus, French Politics and Society, 16, 1, 23-37.

FAUQUet J.M., Hennion A., 2000, La grandeur de Bach. L'amour de la musique en France au XIX ${ }^{e}$ siècle, Paris, Fayard.

FAURE C., 1989, Le projet culturel de Vichy. Folklore et révolution nationale, 1940-1944, Lyon, Presses Universitaires de Lyon.

FAURE M., 1997, Du néoclassicisme musical dans la France du premier XX ${ }^{e}$ siècle, Paris, Klincksieck.

FulCher J., 1999a, French Cultural Politics and Music. From the Dreyfus Affair to the First World War, New York, Oxford University Press.

FULCHER J., 1999b, The Composer as Intellectual: Ideological Inscriptions in French Interwar Neoclassicism, The Journal of Musicology, 17, 2, 197-230.

Gergely J., 1980, André Schaeffner, Revue de Musicologie, 66, 2, 255-260.

GÉTREAU F., 1993, Aux origines du Musée de la musique. Les collections inctrumentales du Conservatoire de Paris, 1793-1993, Paris, Kliencksieck.

GÉTREAu F., 2001, Le patrimoine musical de deux musées parisiens, in ChIMÈNES M., (ed.), La vie musicale sous Vichy, Paris, Complexe, Collection «IHTP », 183-199.

GuÉGAN B., 1920-1922, Almanach de Cocagne pour l'an... Dédié aux vrais gourmands et aux francs buveurs, Paris, Éditions de la Sirène.

HeNnion A., 1993, La passion musicale, une sociologie de la médiation, Paris, Métailié.

HÉRITIER-AugÉ F., 1991, Les Musées de l'Éducation nationale. Mission d'étude et de réflexion, Paris, La Documentation Française.

HOÉRÉE A., 1926, En marge du Festival de Zürich. Querelles esthétiques : la Musique, langage proprement national, Revue Pleyel, 35, 17-20.

Humbert A., 2004, Notre guerre. Souvenirs de Résistance : Paris 1940-41, le bagne, occupation en Allemagne, Paris, Tallandier.

InSTITUT D'ETHNOLOGIE ET MUSÉE D'ETHNOGRAPHIE, 1931, Instructions sommaires pour les collecteurs d'objets ethnographiques, Paris, Institut d'Ethnologie (brochure rédigée par LEIRIS M.).

JAMIN J., 1982, Objets trouvés des paradis perdus. À propos de la Mission Dakar-Djibouti, in Hainard J., KHAER R., (eds.), Collections passion, Neuchâtel, Musée d'Ethnographie, 69-100.

JAMIN J., 1986, L'ethnographie mode d'inemploi. De quelques rapports de l'ethnologie avec le malaise dans la civilisation, in HAINARD J., KAEHR R., (eds.), Le mal et la douleur, Neuchâtel, Musée d'Ethnographie, 45-79.

JAMIN J., 1989, Le musée d'ethnographie en 1930: l'ethnographie comme science et comme politique, in RiviÈRe G.H., La Muséologie selon Georges Henri Rivière. Cours de muséologie I textes et témoignages, Paris, Dunod, 110-121.

JAMIN J., 1996, Introduction, in LeIRIS M., Miroir de l'Afrique, Paris, Gallimard, Collection « Quarto », 9-59.

JAMIN J., 1999, Documents revue. La part maudite de l'ethnographie, L'Homme, 151, 257-266.

JAMIN J., WiLliamS P., 2001, Jazzanthropologie, L'Homme, 158-159, 7-28.

JEANNERET A., 1921, Rythmique, La Revue Musicale, 10, 166-167. 
KLEIN J.C., 1990, Swings : La Revue Nègre, in BARROT O., ORY P., Entre deux guerres. La création française, 1919-1939, Paris, François Bourin, 363-377.

KRADER B., 1958, Bibliography, Ethnomusicology, 2, 1, 26-34.

LAMBERT B., 1998, Le metteur en scène et la peau de son comédien, Sociétés et Représentations, 6, 465-483.

LAURIÈRE C., 1999, Paul Rivet, vie et œuvre, Gradhiva, 26, 109-128.

LAURIÈRE C., 2003, Georges Henri Rivière au Trocadéro. Du magasin de bric-à-brac à la sécheresse de l'étiquette, Gradhiva, 33, 57-66

Lebovics H., 1995, La vraie France : les enjeux de l'identité culturelle, 1900-1945, Paris, Belin.

LeIRIS M., 1938, Du Musée d'ethnographie au Musée de l'Homme, Nouvelle Revue Française, 299.

Maisonneuve S., 2003, L'invention d'un medium musical: le disque et la musique classique en Europe (1877-1949). Contribution à l'étude de l'émergence de formes et de pratiques culturelles, thèse de doctorat, Florence, Institut Européen de Florence.

MARCEL-Dubois C., 1941, Les instruments de musique de l'Inde ancienne, Paris, Presses Universitaires de France.

MARITAIN J., 1920, Art et scolastique, Paris, Librairie de l'art catholique.

MARTIN D.C., RouefF O., 2002, La France du jazz. Musique, modernité et identité dans la première moitié $d u X X^{e}$ siècle, Marseille, Parenthèses.

Mauss M., 1947, Manuel d'ethnographie, établi par Denise Paulme, Paris, Payot.

MÉTrauX A., 1928, La Civilisation matérielle des Tupi-Guarani, Paris, Librairie orientale P. Geuthner.

MÉTRAUX A., 1989, De la méthode dans les recherches ethnographiques, Gradhiva, 5, 57-72 (réédité par JAMIN J.).

MétrauX A., Rivière G.H., 1928, Les Arts anciens de l'Amérique, Paris, G. Van Oest (préface de D'HARCOURT R.).

MiLHAUd D., 1924, Jazz-band et instruments mécaniques - Les ressources nouvelles de la musique, L'Esprit Nouveau, 25, n.p.

Montandon G., 1919, La Généalogie des instruments de musique et les cycles de civilisation, suivi de Catalogue raisonné des instruments de musique du Musée d'ethnographie de Genève, Genève, Albert Kundig.

MulLer B. , 2003, Lucien Febvre, lecteur et critique, Paris, Albin Michel.

NiETZSCHE F., 1957, Lettres à Peter Gast, Introduction et notes de SCHAEFFNER A., Monaco, Éditions du Rocher (2 volumes).

Paulme D., 1992, Lettres de Sanga à André Schaeffner, suivi des Lettres de Deborah Lifchitz et Denise Paulme à Michel Leiris, Paris, Fourbis.

PAulme-SchaefFner D., 1982a, André Schaeffner 1895-1980, Revue de Musicologie, Numéro spécial «Les fantaisies du voyageur. XXIII variations Schaeffner », 68, 1-2, 362-365.

PAUlme-SchaefFNeR D., 1982b, Les fantaisies du voyageur. XXXIII variations Schaeffner, Revue de Musicologie, Numéro spécial «Les fantaisies du voyageur. XXIII variations Schaeffner », 68, 1-2, 401-408.

PORCILE F., 2001, Les conflits de la musique française (1940-1965), Paris, Fayard.

RiEMANN H., 1931 (1882 pour la lère édition allemande), Dictionnaire de musique (traduction française par Humbert G.), 3e édition entièrement refondue et augmentée, SCHAEFFNER A., (ed.), collab. Pincherle M., RoKseth Y., Tessier A., Paris, Payot.

Rivière G.H., 1989, La Muséologie selon Georges Henri Rivière. Cours de muséologie / textes et témoignages, Paris, Dunod.

Rivière J., 1911, Études. Baudelaire, Paul Claudel, André Gide, Rameau, Bach, Franck, Wagner, Moussorgsky, Debussy, Ingres, Cézanne, Gauguin, Paris, NRF. 
Ronsard P. De, 1924, Poésies choisies, publiées par Sorg R. et GuÉGAn B., et suivies de chœurs de Costeley, Orlande de Lassus et Janequin transcrits par Schaeffner A., Paris, Payot.

RouefF O., 2006, Politiques d'une «culture nègre ». La Revue Nègre comme événement public, Anthropologie et Sociétés (à paraître).

Rouget G., 2001, Le Département d'ethnomusicologie du Musée de l'Homme, L'Homme, 171-172, 513-524.

SACHS C., 1934, La signification, la tâche et la technique muséographique des collections d'instruments de musique, Mouseion, 27-28, 5-36.

SACHS C., 1938, Les instruments de musique de Madagascar, Paris, Travaux et mémoires de l'Institut d'Ethnologie.

SAPIRO G., 1999, La guerre des écrivains, 1940-1953, Paris, Fayard.

SCHAEFFNER A., 1922a, De quelques préjugés anti-wagneriens, Le Ménestrel, 10 et 17 novembre, 445447 et $457-459$.

SCHAEFFNER A., 1922b, Sur quelques caractères de l'influence franckiste, La Revue Musicale, 2, 142 154.

SCHAEFFNER A., 1923a, Concerts Koussevitzky (18 octobre), Le Ménestrel, 26 octobre.

SCHAEFFNER A., 1923b, Richard Wagner et l'opéra français du XIX ${ }^{\mathrm{e}}$ siècle, La Revue Musicale, 12, 111-131.

SCHAEFFNER A., 1924a, La musique et la Renaissance en France. À propos des fêtes de Ronsard, Le Courrier Musical, $1^{\mathrm{er}}$ avril, 189-191.

SCHAEFFNER A., 1924b, Quelques remarques autour de Ronsard et de la «Chanson Française », La Revue Musicale, 5, 65-74.

SCHAEFFNER A., 1925, Sur Jacques Rivière, La Revue Musicale, 7, 151-159.

SCHAEFFner A., 1926a, Compte rendu de E. Jacque-Dalcroze, Préparation pour une méthode de solfège rythmique vocal basée sur l'expérience des sensations de durée et de dynamisme et sur une éducation des centres nerveux (1er fascicule, Lausanne-Paris, Jobin-Rouart-Lerolle, 1925), La Revue Musicale, 1, 57-58.

SCHAEFFner A., 1926b, Notes sur le musique des afro-américains, Le Ménestrel, 7 parties du 25 juin au 6 août.

SchaefFner A., 1927a, Le clavecin, in La LaURencie L. De, Lavignac A., (eds.), Encyclopédie de la musique et Dictionnaire du Conservatoire, Paris, Delagrave, 2, 3, 2036-2060.

SCHAEFFNER A., 1927b, Wanda Landowska ou le retour aux « humanités » de la musique, La Revue Musicale, 8, 254-278.

SCHAEFFNER A., 1927c, Compte rendu de Albert Jeanneret, Méthode pour le développement de l'œil du musicien (Paris, Senak, 1927), La Revue Musicale, 1, 89-90.

SCHAEFFNER A., 1927d, Compte rendu de La Schola Cantorum, son histoire depuis sa fondation jusqu'en 1925 (Paris, Blond et Gay, 1927), La Revue Musicale, 2, 181.

SCHAEFFNER A., 1929, Des instruments de musique dans un musée d'ethnographie, Documents, 5, 248-254.

SCHAEFFNER A., 1931a, Strawinsky, Paris, Rieder.

SCHAEFFNER A., 1931b, Projet d'une classification nouvelle des instruments de musique, Bulletin du Musée d'Ethnographie du Trocadéro, 1, 21-25.

SCHAEFFNER A., 1932, D'une nouvelle classification des instruments de musique, La Revue Musicale, $11,215-231$.

SCHAEFFnER A., 1934, Parmi les origines corporelles des instruments de musique, Le Ménestrel, 8 , $65-70$.

SchaefFner A., 1935a, Vogue et sociologie du jazz, in Febvre L., (ed.), Encyclopédie Française, tome 16: Arts et littératures dans la société contemporaine (1), Paris, Comité pour l'Encyclopédie Française, 16.72.11-16.72.13. 
SchAefFner A., 1935b, Les instruments de musique, in FebVRe L., (ed.), Encyclopédie Française, tome 16: Arts et littératures dans la société contemporaine (1), Paris, Comité pour l'Encyclopédie Française, 16.36.13-16, 16.38.1.

SCHAEFFNER A., 1936, Origine des instruments de musique. Introduction ethnologique à l'histoire de la musique instrumentale, Paris, Payot, Collection « Bibliothèque musicale ».

Schaeffner A., 1948, Pré-théâtre, Polyphonies, 1, 7-17 (repris in Schaeffner, 1998, 21-31).

SChAEFFNER A., 1950, La découverte de la musique noire, Présence Africaine, 8-9, 205-218.

SCHAEFFNER A., 1956 (1954), Ethnologie musicale ou musicologie comparée ?, Les colloques de Wégimont, Bruxelles, Elsevier, 18-32.

SCHAEFFner A., 1967, Allocution pour le cinquentenaire de la SFM, Revue de Musicologie, 53, 2, 103-109.

SCHAEFFNER A., 1980 (1964), Discours de départ à la retraite, in JAMIN J., André Schaeffner, Objets et Mondes. La revue du Musée de l'Homme, 20, 3, 131-135.

SCHAEFFNER A., 1980, Essais de musicologie et autres fantaisies, Paris, Le Sycomore.

SCHAEFFNER A., 1990, Le sistre et le hochet. Musique, théâtre et danse dans les sociétés africaines, Paris, Hermann.

SCHAEFFNER A., 1996 (1931), Le beau musical, Gradhiva, 20, 95-107 (textes établis, présentés et annotés par JAMIN J.).

SCHAEFFNER A., 1998a, Variations sur la musique, Paris, Fayard.

SCHAEFFNER A., 1998b (1942-1989), Musique savante, musique populaire, musique nationale, in SCHAEFFNER A., Variations sur la musique, Paris, Fayard, 55-80.

SCHAEFFner A., 1998c (1951), Musique populaire et art musical, in SchAEFFNER A., Variations sur la musique, Paris, Fayard, 32-54.

SCHAEFFner A., 2006, Introduction à Musique et danses funéraires chez les Dogons de Sanga, L'Homme, 177-178 (à paraître).

SCHAEFFNER A., SChlOezer B. DE, 1925, Les courants de la musique russe contemporaine, in LA Laurencie L. De, Lavignac A., (eds.), Encyclopédie de la musique et Dictionnaire du Conservatoire, Paris, Delagrave, 1, II, 159-175.

SCHLOEZER B. DE, 1922, Arnold Schoenberg : Pierrot Lunaire (concerts Wiener), La Revue Musicale, $5,267-269$.

Schloezer B. De, 1927, Compte rendu de Ceeuroy A., Schaeffner A., Le Jazz (1926, Paris, Aveline), La Revue Musicale, 7, 581-582.

SCHLOEZER B. DE, 1929, Igor Stravinsky, Paris, Éditions Aveline, Collection «La Musique Moderne ».

SCHWERKe I., 1926, Le Jazz est mort ! Vive le Jazz !, Le Guide du Concert, 12-19 mars.

SEGalen M., 2005, Vie d'un musée 1937-2005, Paris, Stock.

SERRY H., 2004, Naissance de l'intellectuel catholique, Paris, La Découverte.

SHERMAN D., 2004, Objects Ethnographic. Objects, Museum and the Colonial Inheritance of French Ethnography, French Historical Studies, 27, 3, 669-703.

Sibeud E., 1997, «L'Afrique d'une société savante » : les africanistes et leur mémoire, in PIRIOU A., Sibeud E., (eds.), L'africanisme en questions, Paris, Centre d'Études Africaines-EHESS, 71-88.

SIBEUD E., 2002, Une science impériale pour l'Afrique? La construction des savoirs africanistes en France, 1878-1930, Paris, Éditions de la Maison des Sciences de l'Homme.

Singleton E., 1922, États-Unis d'Amérique, in La Laurencie L. DE, LavignaC A. DE, (eds.), Encyclopédie de la musique et Dictionnaire du Conservatoire, Paris, Delagrave, 5, I, 3245-3332.

SociÉté FranÇAise De Musicologie, 1917, Avis aux lecteurs, Bulletin de la SFM, 1 (article téléchargeable sur le site Internet : http://www.sfm.culture.fr/sfm/includes/docts.htm).

VAnhulst H., 2000, La musicologie, in Pistone D., (ed.), Musiques et musiciens à Paris dans les années 1930, Paris, Librairie Honoré Champion, 411-420. 
WeBER F., 2000, Le folklore, l'histoire et l'État en France (1937-1945), Revue de Synthèse, 3-4, 453467.

WEBER F., 2002, L'ethnologie et l'État en France, des années 1930 aux années 1950, communication pour le Groupe de travail «Histoire des politiques du patrimoine ».

WeBER M., 1998 (1924), Sociologie de la musique. Les fondements rationels et sociaux de la musique (traduit et annoté par Molino J. et PedLer E.), Paris, Métailié.

Weber W., 1975, Music and the Middle Class. The Social Structure of Concert Life in London, Paris and Vienna (1815-1848), London-Canberra-New York, Croom Helms-Holmes \& Meyer. 